\title{
Droplet Shape Control Using Microfluidics and Designer Biosurfactants
}

Yuan $\mathrm{Gao}^{1}$, Chun-Xia Zhao ${ }^{1 *}$, Frank Sainsbury ${ }^{1,2,3 *}$

${ }^{I}$ Australian Institute for Bioengineering and Nanotechnology, University of Queensland, St Lucia, QLD, 4072, Australia.

${ }^{2}$ Centre for Cell Factories and Biopolymers, Griffith Institute for Drug Discovery, Griffith University, Nathan, QLD, 4111, Australia.

${ }^{3}$ Synthetic Biology Future Science Platform, Commonwealth Scientific and Industrial Research Organization (CSIRO), Brisbane, QLD 4001, Australia.

KEYWORDS: microdroplet, shape control, aspect ratio, interface, biosurfactant, microfluidics.

\begin{abstract}
Many uses of emulsion droplets require precise control over droplet size and shape. Here we report a 'shape-memorable' micro-droplet formulation stabilized by a polyethylene glycol (PEG)-modified protein-surfactant, the droplets are stable against coalescence for months and can maintain nonspherical shapes for hours, depending on the surface coverage of PEGylated protein. Monodisperse droplets with aspect ratios ranging from 1.0 to 3.4 were controllably synthesized with a flowfocusing microfluidic device. Mechanical properties of the interfacial protein network were explored to elucidate the mechanism behind the droplet shape conservation phenomenon. Characterization of the protein film revealed that the presence of a PEG layer at interfaces alters the mechanical responses of the protein film, resulting in interfacial networks with improved strength. Taking advantage of the prolonged stabilization of non-spherical droplets, we demonstrate the possibility of functionalization of the droplet interface with accessible biotin moieties. The stabilization of microdroplet shape with surface-active proteins that also serve as an anchor for integrating functional moieties, provides a tailorable interface for diverse applications.
\end{abstract}




\section{INTRODUCTION}

Micro-droplets are compartmentalized fluids in tiny volumes $(\sim 0.5 \mathrm{fL}$ to $523.6 \mathrm{~nL}$ for droplets with diameters of 1-1000 $\mu \mathrm{m}$ ), which serve a multitude of uses in material science and industrial applications. ${ }^{1}$ Their compartmentalized structure makes them ideal for carrying therapeutics or cosmetic ingredients and encapsulating bioactive entities such as cells, proteins, enzymes, and nucleic acids for the construction of biomimetic systems. ${ }^{2-3}$ Micro-droplets also hold great promise in chemical and biochemical applications as high-efficiency micro-reactors ${ }^{4-5}$ or as bio-sensing devices. The isolation of reagents inside microdroplets minimizes cross-contamination or interference, resulting in higher sensitivity and accuracy. ${ }^{6}$ In addition, control over droplet behaviours such as motion, merging, splitting, and sorting further expands the uses of droplets towards more flexible settings. For example, many reports demonstrate the utilization of droplets for various types of analysis and biological assays such as DNA amplification and analysis, protein crystallization, biomarker detection, catalysis and kinetic monitoring. ${ }^{7-10}$ Micro-droplets are not only useful by themselves; they could also be applied as well-defined templates for engineering a wide range of structures, such as growing nanocrystal (gold, silver, platinum) dispersions, ${ }^{11}$ assembling microcapsules $^{12}$ and micro-particles. ${ }^{13-15}$

The ultimate success of these applications depends critically on the availability of droplet dispersions with a well-controlled shape and size distribution. Non-spherical shapes may offer many additional benefits due to their anisotropic architectures and larger surface area compared to their spherical counterparts. The properties conferred by shape could be exploited to build materials with unique structures or to build stimuli-responsive systems that exhibit novel responses to external stimulations e.g. hydrodynamic, electrical, optical, ${ }^{16}$ acoustic, ${ }^{17}$ or magnetic-responsive. ${ }^{18-19}$ There is a direct geometrical influence of shape regarding light scattering, ${ }^{20}$ refraction, dichroism, and plasmon resonance phenomenon ${ }^{11}$ and non-spherical shapes pack more densely compared to spherical ones, which would be highly advantageous for applications requiring compact arrangements. However, the controlled synthesis of non-spherical droplets in large quantities remains difficult. To date, the production of micro-droplets has been dominated by spherical droplets because of the tendency towards fluid equilibration and minimization of interfacial free energy, whether they are formed through conventional bulk emulsification techniques or by shearing flows in microfluidic systems. ${ }^{21}$ 
Current strategies to form non-spherical droplets include manipulating droplets using open patterned surfaces of different wettability. ${ }^{22-23}$ Hancock et al. have shown patterning water droplets by selectively altering the wettability of surfaces, ${ }^{24}$ however, this method is limited by the complexity of precisely creating distinct surface properties at microscale. Another strategy is through interfacial tension driven evolution of droplet morphology where the local accumulation of surface-active emulsifiers at interfaces can partially change the droplet curvature. ${ }^{25-26} \mathrm{~A}$ widely used approach is microchannel confinement, which offers unrivalled performance for the controlled generation of droplets of varying size, shape, morphology, internal structure and chemistry. ${ }^{8,27}$ Using microfluidics to control local flows, the flexible design of microscale geometries and the applicability of auxiliary forces allow a high degree of control over the manipulation of individual droplets. However, for most droplets generated in microfluidic devices, non-spherical shape is short-lived due to the interfacial minimum free energy effect. In order to preserve non-spherical shapes, droplets must stay in the confined conditions or be cured into solid state before they relax back to spherical. Several in situ curing strategies have been reported, for example, by photo-polymerization, ${ }^{28}$ flow lithography, solvent evaporation-induced solidification, ${ }^{29}$ thermal-controlled crosslinking and gelling. ${ }^{28}$ While this approach has enabled the stabilization of capsules and solid particles of multiple shapes (ellipsoids, plugs, rods, disks, cubes, threads, core-shell, Janus), ${ }^{27-28,30}$ it is not favourable for continuous synthesis process due to easily clogging of microfluidic channel by aggregated solids.

In this work, we demonstrate a microfluidic-based method for fabricating non-spherical droplets stabilized by a surface-active peptide and modified protein co-surfactant. AM1 (Ac-MKQLADS LHQLARQ VSRLEHA-CONH 2 ) is a designed peptide with good emulsifying ability; rapidly adsorbing onto oil/water $(\mathrm{O} / \mathrm{W})$ interfaces and forming strong cohesive films in the presence of divalent metal ions via histidine coordination. ${ }^{31}$ S28C, a protein composed of repeating AM1 units, ${ }^{32}$ spontaneously integrates onto AM1-covered interfaces, further enhancing the stability of the system and introducing the possibility of surface modification by conjugated functional moieties. ${ }^{33}$ Herein, using this co-surfactant system, we show the controlled synthesis of polyethylene glycol (PEG)modified protein-stabilized droplets with tuneable shape. Mechanical properties of the interfacial protein network were evaluated to understand the mechanism behind the shape-maintenance phenomenon. These non-spherical droplets offer new opportunities for shape-relevant studies, considering the wide interest in non-spherical particle synthesis, controlled release, sensing, and catalysis applications. 


\section{MATERIALS AND METHODS}

\subsection{Materials}

Miglyol ${ }^{\circledR} 812$ oil (density $0.945 \mathrm{~g} \cdot \mathrm{cm}^{-3}$, viscosity $25.1 \mathrm{mPa} \cdot \mathrm{S}$ at $20^{\circ} \mathrm{C}$ ) was provided by Caesar \& Loretz GmbH (Hilden, Germany). Peptide-surfactant AM1 was purchased from GenScript Corporation (Piscataway, NJ, USA). 4-(2-hydroxyethyl)-1-piperazineethanesulfonic acid (HEPES), zinc chloride $\left(\mathrm{ZnCl}_{2}\right)$, tris(2-carboxyethyl)phosphine hydrochloride (TCEP), methoxypolyethylene glycol maleimide (MeO-PEG5K-Mal, $\mathrm{Mn}=5,000)$ and poly(ethylene glycol)[N-(2maleimidoethyl)carbamoyl]methyl ether 2-(biotinylamino)ethane (Mal-PEG5K-biotin, Mn=5,400) were purchased from Sigma-Aldrich, Co. (St. Louis, MO, USA). Alexa Fluor 488 streptavidin conjugates and a lipophilic dye DiI (1-1'-Dioctadecyl-3,3,3',3'-tetramethylindocarbocyanine perchlorate) were purchased from Thermo Fisher Scientific (Waltham, MA, USA). S28C was expressed from Escherichia coli cells and then purified by a chromatography-based method as previously described. ${ }^{33-34}$ Briefly, the purification process composed of three chromatography steps: first, by an immobilized metal affinity chromatography (IMAC) column to enrich the metal-binding S28C; second, removing charged contaminants by sequential cation and anion exchange; third, selection and focusing of the aliphatic S28C by reversed phase chromatography (RPC). All the buffers were produced in lab with fresh Milli-Q water $\left(\sim 18.2 \mathrm{M} \Omega \cdot \mathrm{cm}^{-1}\right)$ and $\mathrm{pH}$ adjusted with hydrochloric acid or sodium hydroxide.

\subsection{S28C purification and PEGylation}

S28C was conjugated with MeO-PEG5K-Mal via thiol-maleimide coupling reaction. The detailed procedures were depicted in a flow chart in Fig. S1(A). First, the lyophilized S28C was dissolved in HEPES buffer ( $25 \mathrm{mM}, \mathrm{pH} 7.0$ ) that containing $4 \mathrm{mM}$ TCEP to reach a protein concentration of 400 $\mu \mathrm{M}$. Then, MeO-PEG5K-Mal (or Mal-PEG5K-biotin) powder was weighed directly in a $5 \mathrm{~mL}$ reaction tube. S28C solution was added into the tube drop-by-drop while stirring with a magnetic stir bar. The protein S28C and MeO-PEG5K-Mal were mixed at a molar ratio of 1:20 (S28C: MeOPEG5K-Mal) and stirred at $250 \mathrm{rpm}$ under $4{ }^{\circ} \mathrm{C}$ overnight $(16 \mathrm{~h})$. The reacted sample mixtures were diluted to $10 \mathrm{~mL}$ to retard the reaction and the mixtures were ran through an IMAC column to isolate the PEG-conjugated S28C. Reversed phase high performance liquid chromatography (RP-HPLC) analysis and sodium dodecyl sulfate-polyacrylamide gel electrophoresis (SDS-PAGE, Mini- 
PROTEAN® TGX ${ }^{\mathrm{TM}}$ Gels, Bio-Rad, CA, USA) were used to verify the PEGylated S28C (denoted as S28C-PEG) could be separated from other substrates by IMAC, as shown in Fig. S1(B). Fig. S1(C) showed the PEGylation and Biotinylation reaction products (Lane 2 and 5) and corresponding purified protein (Lane 4 and 7). The PEGylation reaction was then optimized using a molar ratio of 1:40 (S28C: MeO-PEG5K-Mal), followed by continuously stirring under room temperature for 3 hours. To further remove the remaining free PEG and salts, IMAC eluates were dialyzed against 25 mM HEPES (pH 7.5) overnight and the dialyzed samples were collected and lyophilized. The S28CPEG used in this work were 1:40 conjugated proteins unless stated otherwise.

\subsection{Fabrication of microfluidic chips}

Microfluidic chips were fabricated based on standard SU-8 photolithography and soft lithography techniques. ${ }^{35}$ Briefly, PDMS and curing agent were weighed at a mass ratio of 10:1 and mixed vigorously using a centrifugal mixer. Then polymer mixture was poured onto the previously designed silicon wafer master with the desired channel pattern, degassed and incubated under $80{ }^{\circ} \mathrm{C}$ for 20 min to cure the polymer before cutting out the cured PDMS slab. Glass slides were cleaned with ethanol and Milli-Q water, dried by nitrogen gas, then coated with a thin layer of PDMS using a spin coater. Oxygen plasma treatment was performed on both PDMS surfaces to be bonded (the channel slab and the slide), which were then quickly pressed together before incubation in $80{ }^{\circ} \mathrm{C}$ oven for another $20 \mathrm{~min}$. Oxygen plasma treatment is a critical step, converting the inherently hydrophobic PDMS surface to a hydrophilic surface. The hydrophobicity of PDMS would recover with time, so the chip has to be used right after fabrication. The chip channel was immediately fully rinsed with Milli-Q water after taking out from the oven to maintain its hydrophilicity. The chip was rinsed with $0.5 \mathrm{mM}$ EDTA between repeated runs, washed with $\sim 15 \mathrm{~mL}$ Milli-Q water, which was expelled before use.

\subsection{Microfluidic production of oil-in-water droplets}

A flow focusing microfluidic device with three inlets was used for droplet generation and control (refer to Fig. 1A). The spiral channel has a width of $200 \mu \mathrm{m}$ and a depth of $150 \mu \mathrm{m}$ and a total length of $\sim 0.48 \mathrm{~m}$ to allow sufficient time for the adsorption of protein surfactants on the droplet surface. ${ }^{36-}$

${ }^{37}$ Miglyol 812 was used as the dispersed phase; surface-active peptide AM1 and protein S28C/S28C-PEG were dissolved in $25 \mathrm{mM}$ HEPES buffer ( $\mathrm{pH} 7.0)$ and used as the continuous 
phase. All peptide/protein solutions used in the microfluidic experiments contain $\mathrm{ZnCl}_{2}$ at twice the concentration of AM1 and eight times that of S28C/S28C-PEG. The prepared solutions were fed to the micro-channels by three digitally controlled syringe pumps (Harvard Apparatus PHD 2000, USA). Several flow rate combinations were tested to control the hydrodynamic flow. Droplets were collected in $1.5 \mathrm{~mL}$ Eppendorf tubes for storage or directly onto a glass slide for microscopy. Biotinfunctionalized droplets were also prepared following a same microfluidic protocol using S28C-PEGbiotin. Droplets (produced with $50 \mu \mathrm{M}$ AM1 and $20 \mu \mathrm{M}$ S28C-PEG-Biotin in the presence of $\mathrm{ZnCl}_{2}$ ) were collected at a flow rate of 2:3:3 $\mu \mathrm{L} / \mathrm{min}$ and maintained 1:1 in fresh $50 \mu \mathrm{M}$ AM1 solution containing $100 \mu \mathrm{M} \mathrm{ZnCl}_{2}$ for subsequent functionalization with Alexa fluor@ 488 streptavidin (50 $\mu \mathrm{g} / \mathrm{mL}$ ) for 1 hour at room temperature.

2.5 Microscopy and image analysis

A Nikon optical microscope (Eclipse TS100 Inverted Microscope, Nikon Inc., Japan) equipped with a digital camera (Powershot A640, Canon Inc., Tokyo, Japan) was used to capture the droplets and record videos and images. The size distribution of the micro droplets was analyzed by ImageJ (National Institute of Health, USA). Images were colour-threshold treated to detect of contour of droplets and the size and distribution were measured by the 'particle analyze' function. To compare the dynamic breakup process under different surfactant solution conditions, videos were recorded, and the droplets formed were tracked and measured by a written MATLAB (version R2019a, MathWorks, Inc., USA) script. Fluorescent images of droplets were obtained with a Nikon Eclipse Ti Series Microscope (Nikon Inc., Japan) at an excitation wavelength of $560 \mathrm{~nm}$. For biotinfunctionalized droplets, confocal images were captured with a Leica TSC SP8 Confocal Microscope (Leica Microsystems CMS GmbH, Mannheim, Germany). DiI and Streptavidin Fluro Alexa 488 were excited at $560 \mathrm{~nm}$ and $499 \mathrm{~nm}$, and emission bands were collected between 568-687 $\mathrm{nm}$ and 504-549 nm, respectively.

2.6 Characterization of peptide/protein films by Cambridge interfacial tensiometry (CIT)

Cambridge interfacial tensiometry (CIT) was used to characterize the interfacial network. ${ }^{38} 5 \mu \mathrm{M}$ $\mathrm{S} 28 \mathrm{C} / \mathrm{S} 28 \mathrm{C}-\mathrm{PEG}$ with $40 \mu \mathrm{M} \mathrm{ZnCl}_{2}$ in HEPES buffer $(25 \mathrm{mM}, \mathrm{pH} 7.0$ ) were prepared, $6.5 \mathrm{~mL}$ of each solution was used to fill the CIT sample trough. Proteins may self-assemble into a cohesive network at air-water interface between the two T-shaped bars plunged on the solutions surface, one 
T-Bar was connected to a force sensor, which allows for the detection of stresses required to stretch the protein network. The interfacial structure was subject to periodically expansion and contraction cycle every 1 minute over a 60 min period with a strain up to 300\% (with a constant strain rate of $10 \%$ per second). The stress curve as a function of the displacement of strain was recorded, and elastic modulus was calculated according to the slope of the stress-strain curve. $20 \mu \mathrm{M}$ AM1 solution with $40 \mu \mathrm{M} \mathrm{ZnCl} 2$ was also tested as a comparison group.

\subsection{Interfacial tension and droplet contraction}

The interfacial tension (IFT) for surfactant enriched O/W interfaces were measured by a pendant drop method using a KRÜSS DSA-10 droplet-shape analysis (DSA) instrument. Peptide/protein solutions were prepared with $25 \mathrm{mM}$ HEPES buffer ( $\mathrm{pH} 7.0)$ and then $6.5 \mathrm{~mL}$ of it was filled into a quartz cuvette container. Miglyol oil was supplied through a J-shaped stainless-steel capillary, an oil droplet (about 8-10 $\mu \mathrm{L}$ in volume) immersed in the protein solution was then generated at the capillary tip. The droplet shape variation over time was automatically detected and recorded, and dynamic IFT data were collected. After aging for $20 \mathrm{~min}$, a sudden contraction was performed on the droplet, and the changes of droplet interfacial morphologies were monitored over a 15 min period. 50 $\mu \mathrm{M}$ SDS solution was prepared with Milli-Q water. The IFT profiles were smoothed with the Padé approximation fitting ${ }^{39}$ in GraphPad Prism 8 software (GraphPad, San Diego, CA) for better visualization.

\subsection{Rheological studies and data processing}

A microfluidic-based method was adopted to investigate rheological properties of interfacial accumulated elastic peptide/protein networks. A simple two-inlet microfluidic device (Fig. S2) was used, with Miglyol oil as the dispersed phase (flow rate $\mathrm{Q}_{\mathrm{d}}$ changed from $0.4,1$ and $2.5 \mu \mathrm{L} / \mathrm{min}$ ), and six different surfactant solutions as the continuous phase (flow rate $Q_{c}$ was set to be 4, 8, 16, 32, 64 $\mu \mathrm{L} /$ min for each $\mathrm{Q}_{\mathrm{d}}$ ). Small molecule surfactant sodium dodecyl sulfate (SDS) $(50 \mu \mathrm{M})$, which does not form an interconnected network upon adsorption at oil/water interface, was selected as a control. The breakup of microfluidic oil stream in response to varying shearing conditions were compared and quantitatively analyzed by fitting droplet size (as a function of $Q_{d} / Q_{c}$ ) to a linear-relationship mathematical model (Eq.1). Slopes of the fitted lines were extracted as an elasticity-related factor 
and introduced into a modified model. A MATLAB script was created to determine the optimal parameter that gave the best fitting to the droplet size using the proposed model (refer to Fig. S3).

\section{RESULTS AND DISCUSSION}

\subsection{Conservation of deformed droplet shape by PEGylated biosurfactant}

In biomedical applications, PEGylation is a widely used strategy for drug-carrier systems to improve the bioavailability and provide immune evasion. Shielding by the immunologically inert PEG layer decreases the possibility of early elimination from circulation by the immune system. ${ }^{40} \mathrm{We}$ designed a surface-active protein S28C, which has a single cysteine residue within the sequence (Fig. 1A) to increase the precision and efficiency of site-specific modifications via sulfhydryl-maleimide crosslinking. ${ }^{32}$ Following the single sulfhydryl-maleimide reaction strategy, we obtained PEGylated S28C (denoted as S28C-PEG, refer to Fig. S1). S28C-PEG and S28C were evaluated as the cosurfactant of AM1 in the same microfluidic (a flow focusing device with a long spiral channel) setting to stabilize the generated oil droplets. $50 \mu \mathrm{M}$ AM1 together with $5 \mu \mathrm{M} \mathrm{S} 28 \mathrm{C} / \mathrm{S} 28 \mathrm{C}$-PEG were tested for the controlled production of Miglyol 812 oil droplets (flow rates 1:4:4 $\mu \mathrm{L} / \mathrm{min}$ for Miglyol oil: AM1 solution: S28C solutions, respectively). Unexpectedly, the S28C-PEG stabilized droplets were found to be 'shape-memorable'; they were able to maintain an ellipsoidal shape following deformation by the microfluidic channel (Fig. 1B(iv)). Although the droplets were all deformed in microfluidic channels due to the confinement of channel dimensions (Fig. 1B(i-ii)), those stabilized by $\mathrm{S} 28 \mathrm{C}$ relax back to spherical immediately after escaping the confinement of channel (Fig. 1B(iii)). Further increasing the $\mathrm{S} 28 \mathrm{C}$ concentration 10 -fold $(50 \mu \mathrm{M})$ does not prevent relaxation to

spherical. To see if the PEGylation modification had a significant impact on the interfacial activity of S28C, interfacial tension kinetics at the oil/protein solution (S28C and S28C-PEG) interface was monitored with a pendant drop shape analyser. For both molecules, the interfacial tension decreases rapidly at the beginning of measurement, dropping from $\sim 31.6 \mathrm{mN} \cdot \mathrm{m}^{-1}$ for pure Miglyol 812 oil/water interface and reaching an equilibrium value of around $12 \mathrm{mN} \cdot \mathrm{m}^{-1}$ at the end of $20 \mathrm{~min}$ monitoring, indicating very efficient adsorption of both proteins (Fig. 1D). Although the IFT decrease for the larger S28C-PEG was slightly slower than that of S28C, they both reached a similar 
equilibrium value. Therefore, PEGylation has a negligible effect on the interfacial activity of S28C protein.
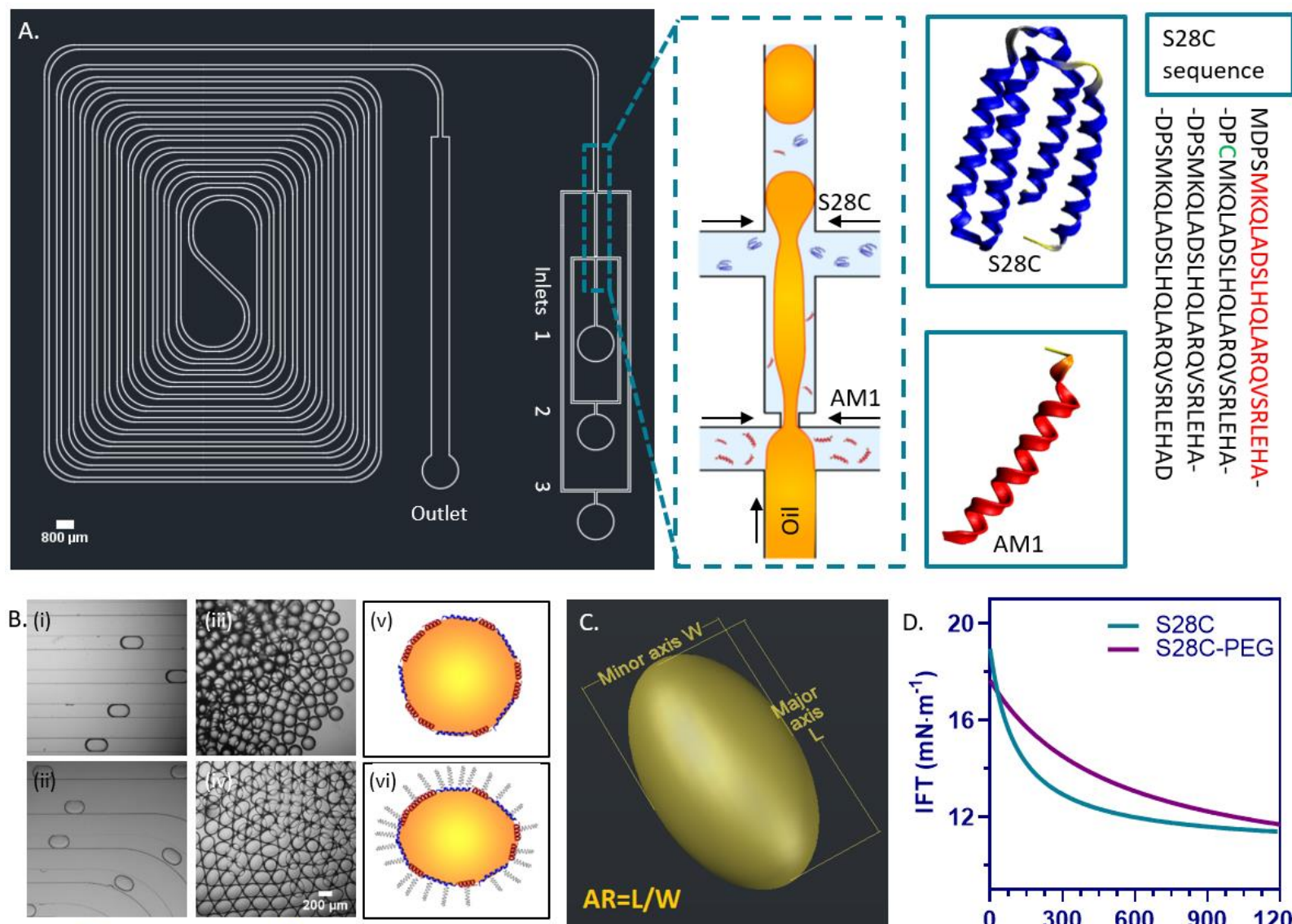

(1) Oil $\omega_{0}$ AM1
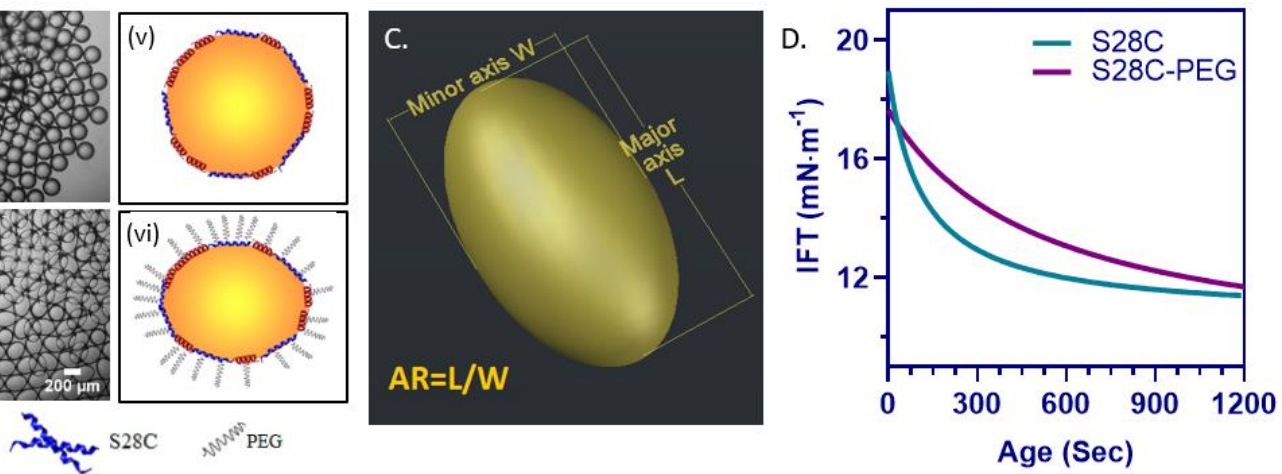

Figure 1. Microfluidic production of peptide/protein stabilized micro-droplets. (A) The microfluidic channel is $150 \mu \mathrm{m}$ in height and the width varies from $200 \mu \mathrm{m}$ for the main channel and $100 \mu \mathrm{m}$ at the junction, scale bar: $800 \mu \mathrm{m}$. Biosurfactants AM1 and S28C were sequentially introduced to produce and stabilize oil droplets. The red-highlighted residues in S28C sequence indicate AM1 sequence, the greenhighlighted residue indicates the cysteine introduced for functionalization. (B) Droplets with different interfacial molecular structures stabilized by: (i) S28C, (ii) S28C-PEG. (iii) and (iv) show corresponding droplet morphologies without the confinement of channel following collection, and (v) and (vi) are corresponding schematics depicting the differences in droplet interfacial structures, scale bar: $200 \mu \mathrm{m}$. S28CPEG was conjugated at a molar ratio of 1:20 (S28C: MeO-PEG5K-Mal). (C) Definition of droplet aspect ratio (AR), where $\mathrm{L}$ represents the droplet length (major axis) and $\mathrm{W}$ stands for the width (minor axis). (D) Comparison of the interfacial tension profile for $5 \mu \mathrm{M}$ of S28C $(\bullet)$ and S28C-PEG ( $\boldsymbol{\Delta})$ at oil-water interface. Both S28C and S28C-PEG were used in the presence of $40 \mu \mathrm{M} \mathrm{ZnCl}_{2}$. 


\subsection{Control over droplet aspect ratio by varying flow rates}

Considering the shape-memorable behaviour of the S28C-PEG stabilized droplets, the opportunity arises to produce long-lasting non-spherical droplets of different aspect ratios (AR; Fig. 1C) by breaking-off the dispersed flow into different lengths when operating within the constraints of microfluidic channel. We tested several flow rate combinations using two concentrations of S28CPEG. The fluid dynamic and break-off of the droplets are dominated by complex interactions of multiple factors, including junction geometry, inertia, viscosity, surface tension etc. ${ }^{41} \mathrm{~A}$ set of dimensionless numbers have been defined to quantify the influence of different factors. ${ }^{42}$ Among the most important are the Capillary number $(\mathrm{Ca})$, which characterizes the relative importance of viscous force over surface tension; flow rate ratio $\mathrm{Q}_{d} / \mathrm{Q}_{c}$, representing the flow rate ratio of the dispersed phase $\left(\mathrm{Q}_{\mathrm{d}}\right)$ and continuous phase $\left(\mathrm{Q}_{\mathrm{c}}\right){ }^{41}$ Models predicting droplet formation and size based on these numbers have been established for certain flow conditions ${ }^{42-46}$ and a power law relationship has been proposed describing the influence of $\mathrm{Q}_{d} / \mathrm{Q}_{\mathrm{c}}$ on resulted droplet size. The relationship can be expressed as:

$$
\frac{d}{d_{c}} \propto\left(\frac{Q_{d}}{Q_{c}}\right)^{\frac{1}{2}}
$$

where $d$ is the droplet size, and $d_{c}$ is the width of channel. A larger $Q_{d} / Q_{c}$ would yield a smaller shearing force thus leading to larger droplets. Since the extent of aspect ratio (AR) variation depends on the droplet size, AR could be controlled simply by changing $\mathrm{Q}_{d} / \mathrm{Q}_{\mathrm{c}}$. Consistent with (Eq.1), when a larger $\mathrm{Q}_{\mathrm{d}} / \mathrm{Q}_{\mathrm{c}}$ was generated by increasing $\mathrm{Q}_{\mathrm{d}}$ from 1 to $5 \mu \mathrm{L} / \mathrm{min}$, elongated droplets were formed. AR ranging from $1.54 \pm 0.04$ to $2.43 \pm 0.18$ were achieved when using $10 \mu \mathrm{M} \mathrm{S} 28 \mathrm{C}-\mathrm{PEG}$ (Fig. $2 \mathrm{~A}(\mathrm{i})$ ). Reducing $\mathrm{Q}_{d} / \mathrm{Q}_{\mathrm{c}}$ by increasing $\mathrm{Q}_{c}$ correspondingly resulted in smaller droplets, with a reduction of AR from > 2 to $\sim 1.7$ as AM1 or S28C feeding rates were increased (Fig. 2A(ii, iii)). However, these droplets showed relaxation to spherical in all but the lowest $Q_{d} / Q_{c}$ after 20 minutes. The observation that the highest surfactant to oil ratio could partially stabilize droplets led us to test higher concentrations of S28C-PEG. 
Increasing the S28C-PEG to $50 \mu \mathrm{M}$, the droplet shape retention was greatly enhanced for all the tested flow rate combinations (Fig. 2A(iv - vi)). Therefore, we inferred that the relaxation rate of droplet shape is inversely proportional to the adsorbed interfacial network. An insufficient coverage of interfacial S28C-PEG network at low bulk solutions would be responsible for the fast relaxation when using $10 \mu \mathrm{M}$ S28C-PEG. The higher supply of S28C-PEG molecules at $50 \mu \mathrm{M}$ results in higher amount of adsorbed S28C-PEG and, presumably, a stronger interfacial network. Notably, when the AM1 flow rate was low, droplets relaxed from $2.06 \pm 0.08$ to $1.22 \pm 0.10$ within 20 min in the presence of $50 \mu \mathrm{M}$ S28C-PEG (Fig. 2A(v)). This indicates a possible synergistic adsorption effect between AM1 and S28C-PEG with insufficient adsorption of AM1 limiting accumulation of S28CPEG at interfaces. For a quantitative comparison of the surfactant coverage at the droplet surface, the droplet volume and the protein solution supplied to each droplet were estimated. As shown in Figs. 2 and S5, droplet shape can be stabilized in the presence of $10 \mu \mathrm{M}$ S28C-PEG when flow combinations result in smaller-AR droplets and higher S28C-PEG concentrations. These data suggest that a higher supply of S28C-PEG to the interface can result in more stable non-spherical droplets. Applying this finding, we further increased the AR of droplets up to 3.4 by decreasing the continuous phase flow rate at $50 \mu \mathrm{M}$ S28C-PEG, resulting in droplets of AR ranging from 2.0 to 3.4 (Fig 2B(i-iii). 

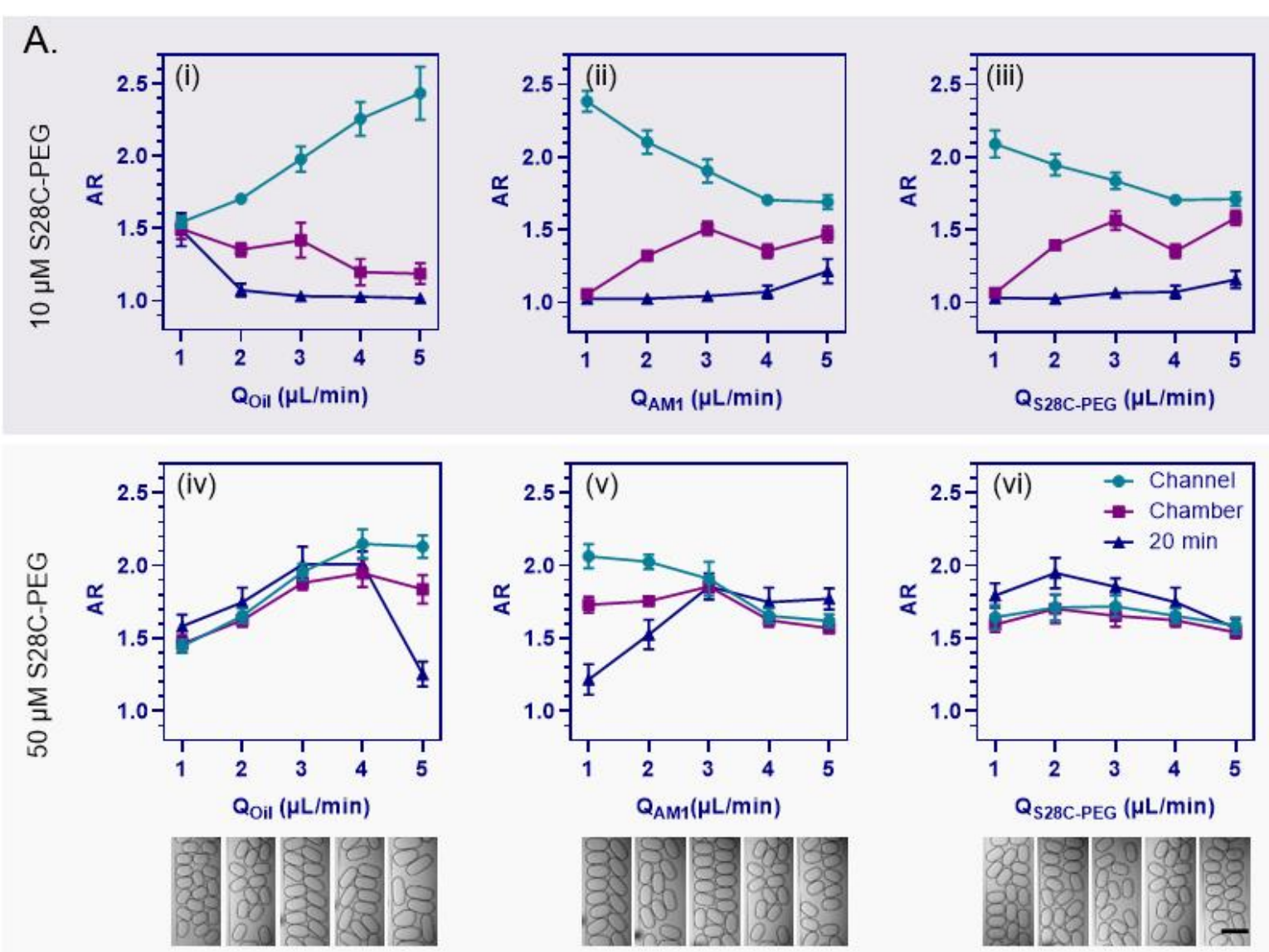

B.
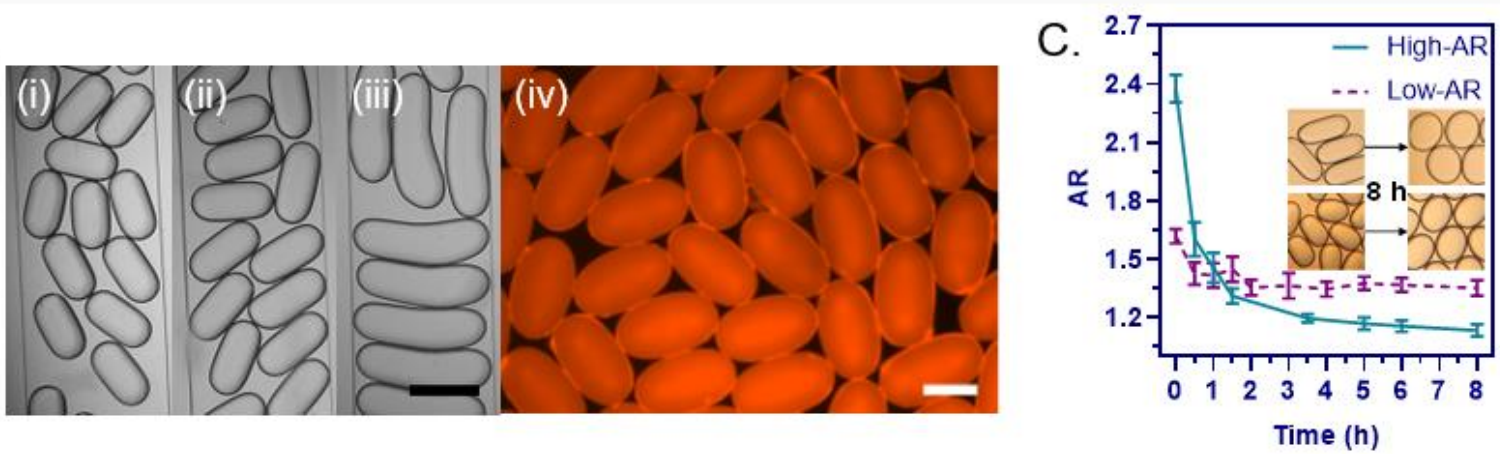

Figure 2. Control over droplet aspect ratio (AR). (A) Droplet AR variation with changing flow rate of (i) oil, (ii) AM1 and (iii) S28C-PEG when using $10 \mu \mathrm{M}$ S28C-PEG, and (iv) oil, (v) AM1 and (vi) S28C-PEG when using $50 \mu \mathrm{M}$ S28C-PEG. The insets show the morphologies of the droplets in the observation chamber. Scale bar: $400 \mu \mathrm{m}$. The flow rates of the labelled phases were changed between 1-5 $\mu \mathrm{L} / \mathrm{min}$ in turn, while keeping the other two phases constant. For AM1 and S28C-PEG solution, the flow rate was fixed at $4 \mu \mathrm{L} / \mathrm{min}$; for oil, the flow rate was fixed at $2 \mu \mathrm{L} / \mathrm{min}$. All peptide/protein solutions contain $\mathrm{ZnCl}_{2}$. Symbols of circle $(\bullet)$, cubic ( $\mathbf{\square})$, and triangle ( $\mathbf{A}$ ) mark AR of droplets that flowing in the channel or collected in the chamber and 20 min post-collection, respectively. (B) Optical microscope images of Miglyol ${ }^{8} 812$ droplets with increasing 
ARs from (i)2.0 to (ii)2.3 to (iii)3.4, and (iv) show Dil-loaded droplets captured by fluorescence microscope. Black scale bar: 400 um, white scale bar: $200 \mu \mathrm{m}$. (C) Droplet stability over an 8-hour observation, the droplets were obtained under flow rates of 1:4:2 $\mu \mathrm{L} / \mathrm{min}$ and 3:9:6 $\mu \mathrm{L} / \mathrm{min}$ (for oil, $50 \mu \mathrm{M}$ AM1 with $100 \mu \mathrm{M}$ $\mathrm{ZnCl}_{2}$, and $10 \mu \mathrm{M}$ S28C-PEG with $80 \mu \mathrm{M} \mathrm{ZnCl}_{2}$ solution), respectively. The insets show the original and final shape over the observation period.

\subsection{Droplet stability against shape relaxation.}

Following collection, droplets with an initial AR of 2.38 \pm 0.07 and $1.62 \pm 0.04$ were monitored for an 8-hour period. The high-AR droplets were almost spherical after $8 \mathrm{~h}$, while the low-AR droplets were more stable, only slightly relaxing during the first hour with a small decrease of AR from $1.62 \pm 0.04$ to $1.42 \pm 0.06$ then remaining stable without further relaxation or coalescence. Similarly stable non-spherical droplets have also been observed for Pickering emulsion droplets stabilized by nanoparticles, and in droplet systems with densely assembled interfacial protein/polymer networks. ${ }^{26}$,

${ }^{47}$ Many of these droplets display shape conservation upon coalescence (a phenomenon termed arrested coalescence), which is related to interfacial jamming of solid particles or high-mechanical modulus molecular networks. ${ }^{26}$ The crowding of these intrinsically robust substances at the interface, reinforces the mechanical strength of the interface, and simultaneously raises steric barriers for molecular motion, turning it from a flexible liquid-like state to a more rigid solid-like state. Here, in an analogous way, the jamming of polymer PEG chains at droplet surface may play a role. The S28C-PEG layer presents a steric constraint at the interface, favouring the dissipation of the relaxation force, effectively preventing the kinetic mass transfer and rebound of the droplets. This coincides with the bulk phenomenon in which including polymer additives into droplet can alter fluid rheology and influence droplet shape rebound. ${ }^{48}$ Bartolo et al. also showed that adding flexible polymers could inhibit droplet rebound on surfaces by generating normal stresses at the interface, which counteracted the surface tension-induced retraction forces. ${ }^{49}$ Consistent with this interpretation, we have previously observed that a proportion of PEG chains in our system lie at the interface. $^{33}$

To compare the PEG surface coverage, the S28C-PEG solution supplied to each resulted droplet (in Fig. 2C) was calculated. While this volume changes from $14.94 \mu \mathrm{L}$ to $7.69 \mu \mathrm{L}$ for the high (2.38) to 
low (1.62) AR droplets, respectively. Considering the higher surface area of high-AR droplet, the surface coverage amounts of S28C-PEG are comparable between the two. The discrepancy of shapedurability is likely, therefore, due to larger relaxation force for the longer shape of the high-AR droplets. For a deformed droplet interface, energy is stored in the droplet system, which is dissipated under relaxation to return to a minimum energy state. The rebound of the deformed droplets depends on surface tension difference-induced relaxation force versus the interfacial structure-derived resistance force. Higher-AR droplets would generate a larger rebound force than shorter ones. Therefore, the PEG layer at the current level of packing density cannot provide enough resistance against this force for the high-AR droplets. Further studies are needed for quantitatively establish the relationship between shape durability and the required minimum PEG surface density.

\subsection{Mechanical properties of interfacial protein network.}

The dynamic mechanical properties of protein films can be characterized by dilational or shear modulus, which quantifies the resistance of material to applied forces. It is described by two components, i.e. an elastic (storage) modulus that quantifies film elasticity against stress, and a viscous (loss) modulus as a measure of energy dissipation accompanying deformation. ${ }^{50-51}$ To explore the impact of PEG-chain on the mechanical properties of interfacial protein network, a tensile test was carried out using a Cambridge interfacial tensiometer (CIT; Fig. 3A). Stress-strain curves are derived for the S28C, S28C-PEG or AM1 protein/peptide networks subjected to 60 min of periodic expansion and compression. At the low-strain region, the response was predominantly elastic for AM1 and S28C. Stress increases rapidly with expending strains at the lower strain levels and then gradually reached a plateau value around $5.63 \mathrm{mN} \cdot \mathrm{m}^{-1}$ (at $\sim 68.7 \%$ strain) for S28C at $5 \mu \mathrm{M}$ and $1.94 \mathrm{mN} \cdot \mathrm{m}^{-1}$ (at $\sim 25.1 \%$ strain) for AM1 at $20 \mu \mathrm{M}$. The plateau value represents the tensile strength of the film ${ }^{52}$ and a larger tensile strength for S28C reflects a stronger film structure compared to AM1. This is despite the fact that the number of AM1 units (four AM1 per S28C) is the same at the concentrations used and may be due to more extensive cross-linking mediated by histidine coordination of $\mathrm{Zn}^{2+} .{ }^{53}$ However, both the two have shown excellent ductile properties that able to resist large strain stretches, as evidenced by the continuous hysteresis curves. The interfacial elastic modulus of the film structure can be calculated from the slope of the initial linear part of the curve, which is described by the equation: ${ }^{53}$ 


$$
\sigma_{s}=E_{s} \cdot \varepsilon
$$

where $\sigma_{\mathrm{s}}$ is the interfacial stress and $\varepsilon$ is the strain. Es is a constant characterizing the twodimensional elastic modulus of the interfacial network. Linear fitting of the data to $5 \%$ strain gives an elastic modulus value of $117.9 \mathrm{mN} \cdot \mathrm{m}^{-1}\left(\mathrm{R}^{2}>0.97\right)$ and $118.3 \mathrm{mN} \cdot \mathrm{m}^{-1}\left(\mathrm{R}^{2}>0.99\right)$ for AM1 and S28C network, respectively. In contrast, for the S28C-PEG enriched interface, no elastic deformation was observed. A possible mechanism is proposed as illustrated in Fig. S4, where the T-bar slips on the PEG layer at the interface. Therefore, only force changes of very small amplitudes were detected, representing the dragging of the force-transducing T-bar over the PEG surface.

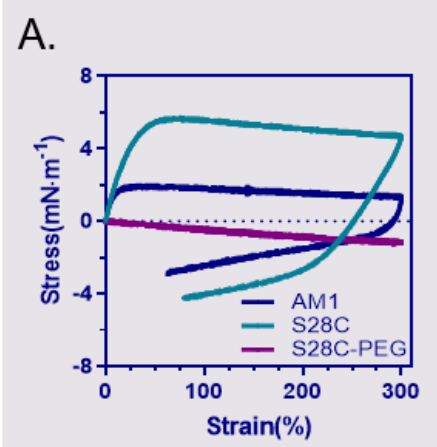

B. (i) SDS vs. AM1

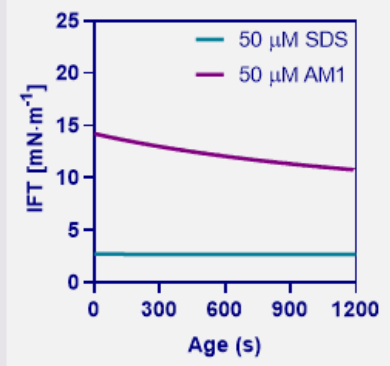

(ii) IFT without AM1

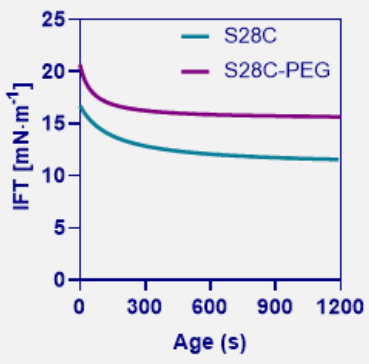

(iii) IFT with AM1

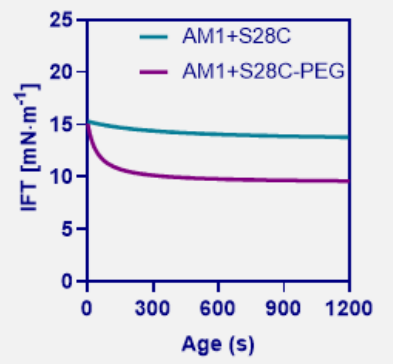

C.
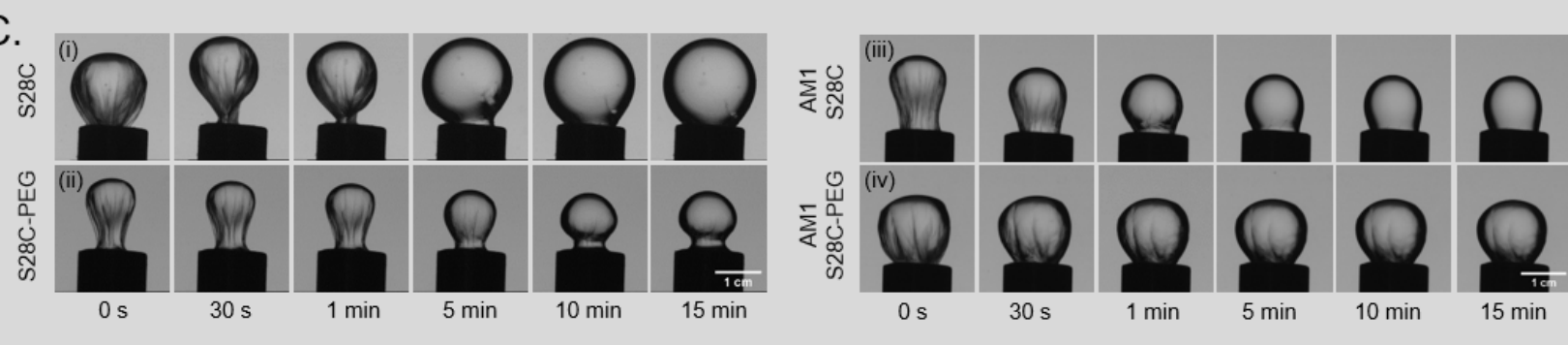

Figure 3. Properties of the interfacial peptide/protein network. (A) Cambridge interfacial tensiometer (CIT)-derived stress-strain hysteresis curves of interfacial surfactant network in respond to continuous expansion and compression. (B) Interfacial tensions (IFT) profile of (i) $50 \mu \mathrm{M}$ SDS vs. $50 \mu \mathrm{M}$ AM1, (ii) 10 $\mu \mathrm{M}$ S28C vs. $10 \mu \mathrm{M}$ S28C-PEG and (iii) $50 \mu \mathrm{M}$ AM1+10 $\mu \mathrm{M}$ S28C mixture vs. $50 \mu \mathrm{M}$ AM1+10 $\mu \mathrm{M}$ S28CPEG mixture over an aging time of $20 \mathrm{~min}$. (C) Monitoring of the surfactant film recovery after sudden contraction, films formed in (i) $5 \mu \mathrm{M} \mathrm{S} 28 \mathrm{C}$; (ii) $5 \mu \mathrm{M} \mathrm{S} 28 \mathrm{C}-\mathrm{PEG}$; (iii) $50 \mu \mathrm{M}$ AM1 co-adsorbed with $10 \mu \mathrm{M}$ S28C and (iv) $50 \mu \mathrm{M}$ AM1 co-adsorbed with $10 \mu \mathrm{M}$ S28C-PEG. The oil droplets were aged for $20 \mathrm{~min}$ in surfactant solutions before a sudden contraction was performed. All peptide/protein solutions used for contraction experiments contain $\mathrm{ZnCl}_{2}$. Scale bar: $1 \mathrm{~cm}$ 
Alternatively, pendant drop tensiometry also offers valuable information about interfacial phenomenon. IFT reduction is a direct quantitative reflection of the interfacial accumulation of surfactants (Fig. 3B). The extremely rapid decreasing and equilibration of IFT for smaller molecule SDS indicates its very fast adsorption and arrangement at $\mathrm{O} / \mathrm{W}$ interface. In contrast, AM1, S28C and S28C-PEG systems showed a gradual decreasing IFT profile, which is probably size dependent, with AM1 adsorbing too fast at $50 \mu \mathrm{M}$ to detect the adsorption curve and resulting in the smallest IFT value of the biosurfactants, $\sim 11 \mathrm{mN} \cdot \mathrm{m}^{-1}$ at $1200 \mathrm{~s}$. However, AM1 and S28C co-adsorption did not show further decreased IFT compared to either of the single component systems. Adsorption may have been reduced by the electrostatic association of S28C (pI 6.5) and AM1 (pI 8.5) under neutral pH. In contrast, AM1 and S28C-PEG co-adsorption system showed a considerably lower IFT value, possibly due to a charge screening effect of PEG.

Following sudden droplet contraction of aged droplets covered with interfacial protein networks, wrinkles can be observed due to the rapid withdrawal of inner liquid. The wrinkle recovery process also manifests the mechanical characteristics (elasticity) of the network ${ }^{54}$. A shown in Fig. 3C, the S28C network was fully recovered after 15 minutes of relaxation, while S28C-PEG still showed wrinkles on the surface and contraction around the neck of the droplet. Similarly, for co-adsorption cases, S28C-PEG significantly delayed the recovery compared with S28C. We speculate that the jammed polymers of the PEG layer partly projecting towards the aqueous phase increases the local interfacial elasticity, posing a larger energy barrier for mass transfer and limiting droplet rebound. Therefore, the S28C-PEG covered interfaces exhibit more rigid features.

\subsection{Rheological investigation at surfactant accumulating interfaces}

Previously, the influence of surfactant elasticity on resulting droplet size was identified and discussed by Zhao et al., finding a significant impact of interfacial elasticity on the dynamic breakup of droplets in microfluidic devices. ${ }^{43} \mathrm{~A}$ dimensionless parameter $\zeta=\sigma / \mathrm{E}$ was introduced to reveal the relative impact of interfacial tension $\sigma$ and interfacial elasticity $\mathrm{E}$, and a correlative relationship between droplet size and $\zeta$-modified $\left(\mathrm{Q}_{\mathrm{d}} / \mathrm{Q}_{\mathrm{c}}\right)^{1 / 2}$ was proposed. ${ }^{43}$ Inspired by this, here we use a microfluidic approach to investigate the rheological response of elastic surfactant network under shearing conditions. Figure 4 displays consecutive snapshots of a complete droplet formationdetachment cycle for different surfactants. With SDS, the oil phase stream was sheared off into droplets at the orifice of the central channel. However, for the peptide/protein systems, 
intermolecular networks increased the local interfacial elasticity, resulting in increased resistance of the oil stream to shearing stress and elongation of the oil stream along the channel (Fig. 4). The extension of the oil stream and delayed break-off phenomenon was extremely obvious for the highly elastic AM1 and S28C-PEG co-adsorbed network (Fig. 4A(vi)), possibly owing to a more closely packed interconnected protein layer and the presence of PEG chains increasing the dissipation of shear stress (Fig. 4B). To quantify the differences in interfacial elasticity, the droplet sizes were analyzed by the power law model (Eq.1). SDS, as a non-elastic control, shows a good fit (Table 1) to this equation, the droplet size follows a linear relationship as a function of $\left(Q_{d} / Q_{c}\right)^{1 / 2}$ under different $\mathrm{Q}_{\mathrm{d}}$ values (Fig. 5). However, for peptide/protein surfactants, although the normalized droplet sizes still have a positive correlation with $\left(\mathrm{Q}_{\mathrm{d}} / \mathrm{Q}_{\mathrm{c}}\right)^{1 / 2}$, the slopes are variable at different $\mathrm{Q}_{\mathrm{d}}$ values (Table 1), which may indicate elasticity differences. This is especially evident for S28C-PEG when $\mathrm{Q}_{\mathrm{d}}$ is lowest, where interfacial network is expected to be dense, much steeper slopes are obtained in the presence and absence of AM1. The slow extrusion of the oil flow allows more time for proteins to accumulate and arrange at the interface, increasing the mechanical strength of the interfacial protein film resulting in larger droplets.
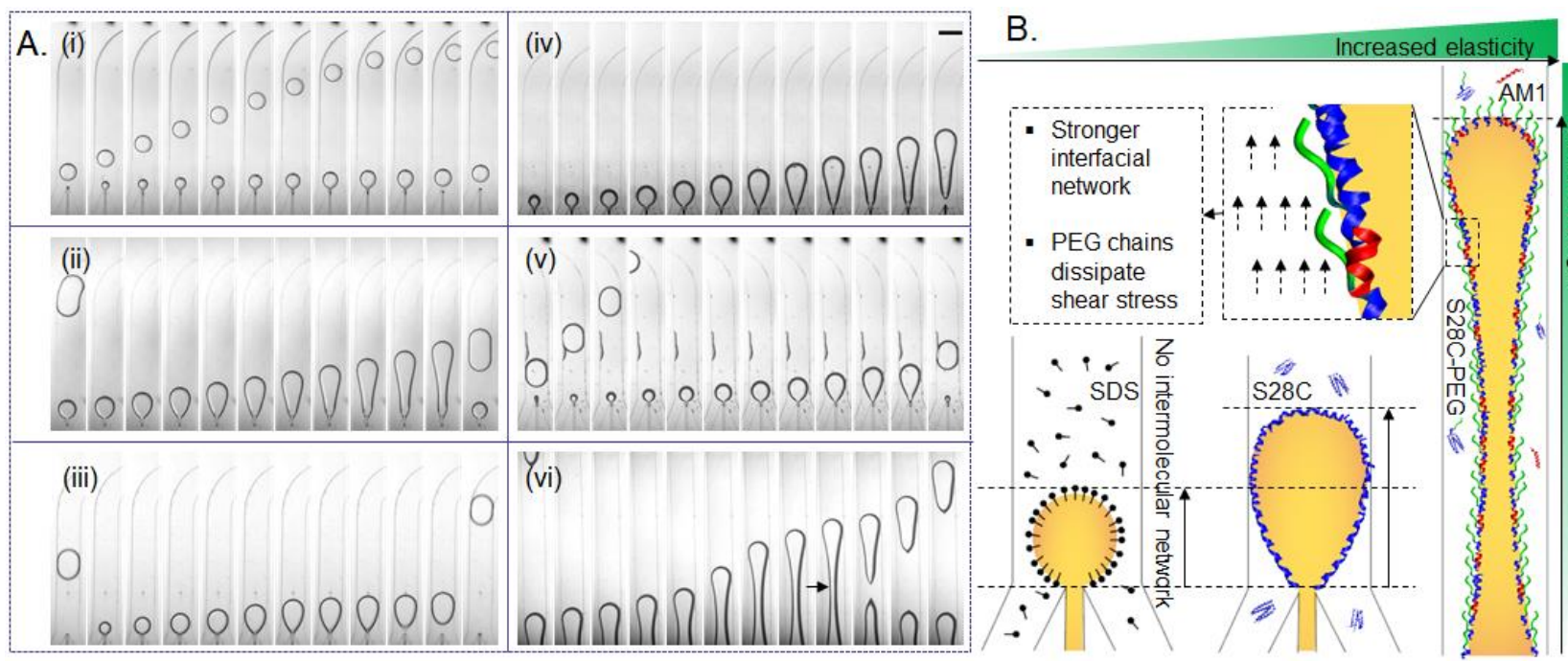

Figure 4. Investigating interfacial elasticity using microfluidics. Consecutive snapshots capturing a complete cycle of droplet formation-detachment process. (A) Oil droplets were formed under the shearing of surfactant solutions of : (i) $50 \mu \mathrm{M}$ SDS, (ii) $50 \mu \mathrm{M}$ AM1, (iii) $10 \mu \mathrm{M}$ S28C, (iv) $10 \mu \mathrm{M}$ S28C-PEG, (v) $50 \mu \mathrm{M}$ AM1 with $10 \mu \mathrm{M}$ S28C, and (vi) $50 \mu \mathrm{M}$ AM1 with $10 \mu \mathrm{M}$ S28C-PEG. All peptide/protein solutions contain 
$\mathrm{ZnCl}_{2}$. Flow rates: $0.4 \mu \mathrm{L} / \mathrm{min}$ for oil and $8 \mu \mathrm{L} / \mathrm{min}$ for all the surfactant solutions. Scale bar: $400 \mu \mathrm{m}$. The arrow in (vi) indicates the break-off position of the oil flow. (B) Schematic showing the differences in interfacial structures before droplet breakup for non-elastic SDS and elastic peptide/protein network-covered interfaces.

A.

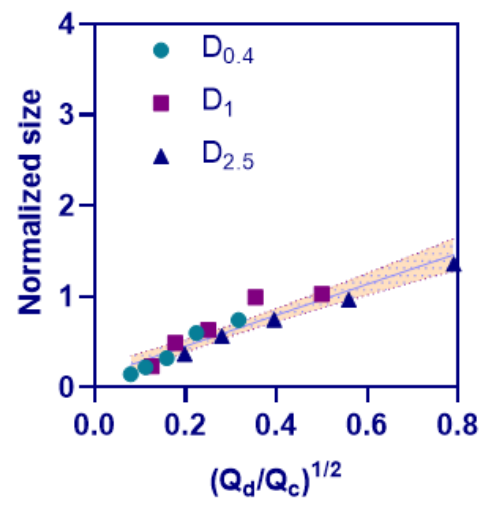

B.

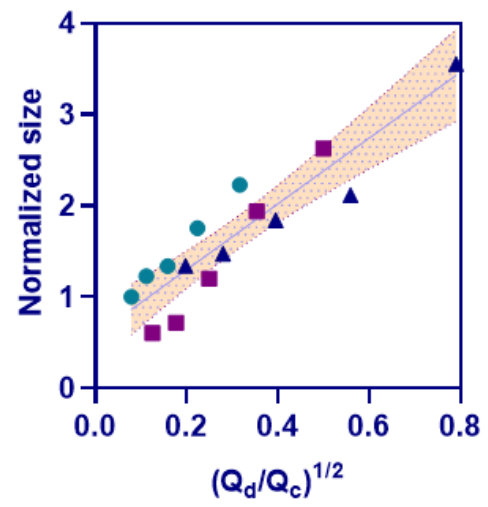

C.

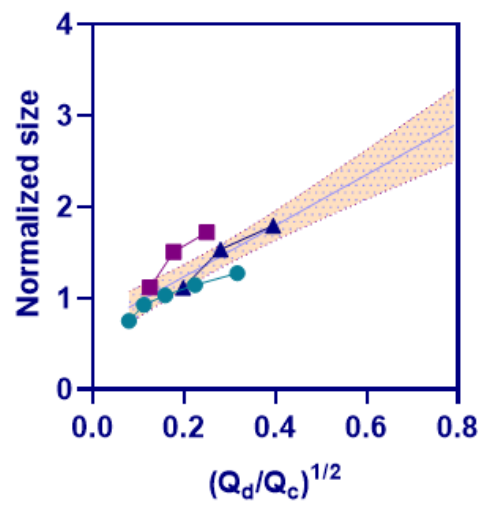

D. $10 \mu \mathrm{M}$ S28C-PEG

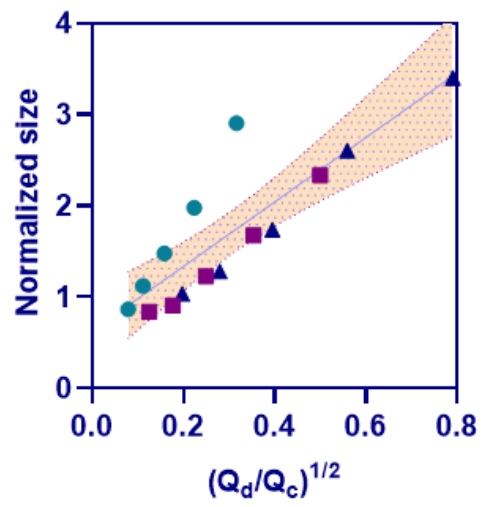

E. $\quad A M 1+S 28 C$

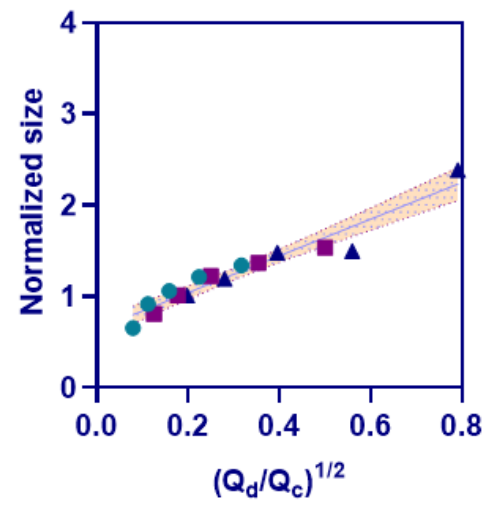

F. AM1+S28C-PEG

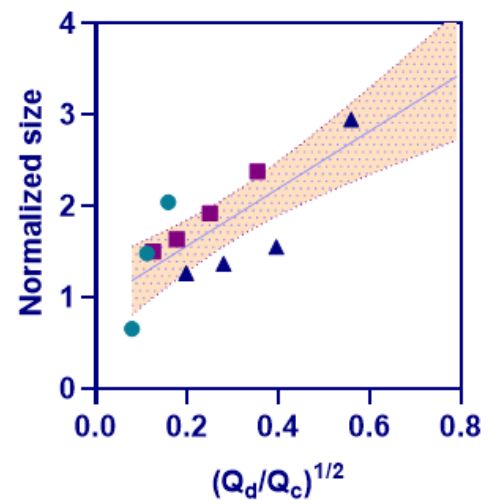

Figure 5. The impact of interfacial elasticity on droplet size. Dependency of the normalized droplets size as a function of $\left(\mathrm{Q}_{\mathrm{d}} / \mathrm{Q}_{\mathrm{c}}\right)^{1 / 2}$ upon varying $\mathrm{Q}_{\mathrm{d}}\left(0.4(\bullet), 1(\boldsymbol{\bullet}), 2.5(\boldsymbol{\Delta})(\mu \mathrm{L} / \mathrm{min})\right.$ and $\mathrm{Q}_{\mathrm{c}}(4,8,16,32,64 \mu \mathrm{L} / \mathrm{min})$ for systems of (A) $50 \mu \mathrm{M}$ SDS, (B) $50 \mu \mathrm{M}$ AM1, (C) $10 \mu \mathrm{M}$ S28C, (D) $10 \mu \mathrm{M}$ S28C-PEG, (E) $50 \mu \mathrm{M}$ AM1 with $10 \mu \mathrm{M}$ S28C, and (F) $50 \mu \mathrm{M}$ AM1 with $10 \mu \mathrm{M} \mathrm{S} 28 \mathrm{C}-\mathrm{PEG}$. All peptide/protein solutions contain $\mathrm{ZnCl}_{2}$.

Normalized droplet size $\mathrm{D}$ is calculated by $\mathrm{D}=\mathrm{L} * \mathrm{~W} / \mathrm{dc}^{2}$. Where $\mathrm{L}, \mathrm{W}$ represents droplet length and width, dc stands for channel width. The straight line in the plots shows the least square linear regression of the data, and the shaded area indicates the $95 \%$ confidential band. 
Table 1. Fitting parameters derived by least square linear regression of normalized droplets size versus varying flow rate ratio.

\begin{tabular}{|c|c|c|c|c|c|c|c|c|}
\hline \multirow[b]{2}{*}{ Systems } & \multicolumn{4}{|l|}{ Slope k } & \multicolumn{4}{|c|}{$\mathrm{R}$ square } \\
\hline & $\mathrm{Q}_{\mathrm{d}}=0.4$ & $\mathrm{Q}_{\mathrm{d}}=1$ & $\mathrm{Q}_{\mathrm{d}}=2.5$ & $\begin{array}{l}\text { All } \\
\text { points }\end{array}$ & $\mathrm{Q}_{\mathrm{d}}=0.4$ & $\mathrm{Q}_{\mathrm{d}}=1$ & $\mathrm{Q}_{\mathrm{d}}=2.5$ & $\begin{array}{l}\text { All } \\
\text { points }\end{array}$ \\
\hline SDS & 2.649 & 2.127 & 1.614 & 1.704 & 0.9718 & 0.8824 & 0.9945 & 0.8987 \\
\hline AM1 & 5.119 & 5.695 & 3.62 & 3.606 & 0.9909 & 0.9896 & 0.9307 & 0.8344 \\
\hline $\mathrm{S} 28 \mathrm{C}$ & 2.052 & 4.684 & 3.365 & 2.801 & 0.9322 & 0.9308 & 0.9464 & 0.8867 \\
\hline S28C-PEG & 8.522 & 4.125 & 4.138 & 3.535 & 0.9956 & 0.9908 & 0.9934 & 0.7392 \\
\hline $\mathrm{AM} 1+\mathrm{S} 28 \mathrm{C}$ & 2.673 & 1.85 & 2.14 & 2.013 & 0.8932 & 0.9310 & 0.9172 & 0.9232 \\
\hline AM1+S28C-PEG & 17.18 & 3.895 & 4.632 & 3.144 & 0.9561 & 0.9907 & 0.8538 & 0.7453 \\
\hline
\end{tabular}

To quantify the impact of accumulating elastic surfactant at interfaces on the breakup behaviour of microfluidic flows, we propose a modified size- $\left(\mathrm{Q}_{\mathrm{d}} / \mathrm{Q}_{\mathrm{c}}\right)^{1 / 2}$ relationship that takes elastic properties into account. Assuming the slope (Table 1) positively correlates with interfacial elasticity, a correction parameter $z=\sigma \cdot E$ is introduced into the original power law equation, where $\sigma$ is the interfacial tension and E stands for the elasticity-related factor calculated from the normalized slope $\mathrm{k} / \bar{k}_{\mathrm{SDS}}$ for each system. $\bar{k}_{\mathrm{SDS}}$ is the averaged slope for SDS (i.e. $\mathrm{E}=1$ for the non-elastic SDS system). Applying this correction parameter $z$, all droplet sizes (Fig. 6A) collapsed to one correlation curve (Fig. 6B), which was expressed by: $\mathrm{D} \propto\left(\mathrm{Q}_{\mathrm{d}} / \mathrm{Q}_{\mathrm{c}}\right)^{1 / 2} \cdot(\sigma \cdot \mathrm{E})^{0.36 \pm 0.09}$. Therefore, the impact of accumulating (shown by reduction of interfacial tension, $\sigma$ ) elastic (reflected by E values) surfactant at a liquidliquid interface on the resulting droplet size is modelled by this equation, showing that forming highelasticity interfacial structures negatively affects the breakup of microfluidic streams leading to larger droplets. This equation provides a simple and direct correlation between droplet size and interfacial elasticity, showing that the droplet size increases with the elasticity, mainly due to the delay of droplet breakup hindered by the elasticity of the interface. 
A. Original data
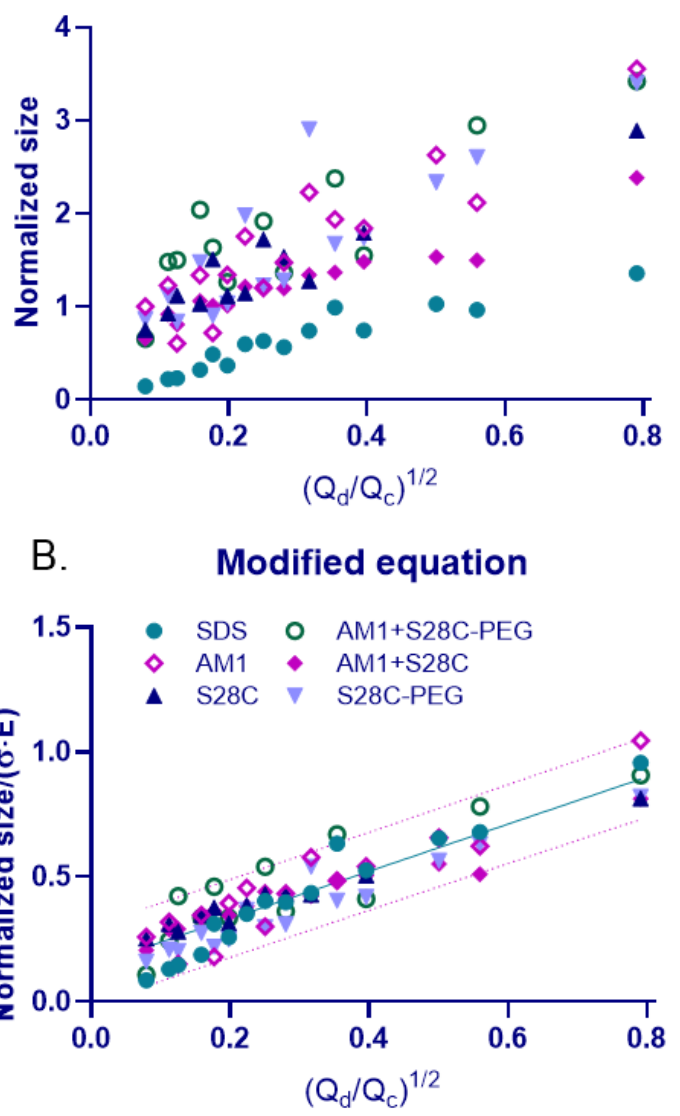

Figure 6. Modified equation to introduce the effects of elasticity on droplet size. (A) Original data were replotted from Figure 5, (B) elasticity factor-modified data. The straight line shows a fitting curve when a correcting factor $(\sigma \cdot E)^{0.36}$ is applied, which can be expressed by equation: $D \propto\left(Q_{d} / Q_{c}\right)^{1 / 2} \cdot(\sigma \cdot E)^{0.36 \pm 0.09}$, where $D$ is the normalized droplet size, $Q_{d} / Q_{c}$ is the flow rate ratio of dispersed phase to continuous phase, $\sigma$ is the interfacial tension, $\mathrm{E}$ is the elasticity-related factor, an exponential parameter within the range of $0.36 \pm 0.09$ all gives a good fitting of droplet size with $\mathrm{R}^{2}>0.80$. The dashed lines show $95 \%$ prediction band.

\subsection{Bio-functionalization of droplet interfaces}

The ability to manipulate droplet shape and their interfacial properties may be of great interests in many biomedical related applications. ${ }^{26,55}$ The protein-covered interfaces presented in this work offer great flexibility for interfacial modification through protein engineering and/or bioconjugation. ${ }^{32-33}$ With a top-down modification strategy, the interfacial properties of protein- 
stabilized droplets can be customized on-demand and, ideally, following their formation. For example, designer peptide/protein adsorbed droplet interfaces can be functionalized with celltargeting ligands to promote its interactions with specific cells. ${ }^{33,56}$ As a demonstration of the possibilities, and of the stability of non-spherical shape retention, we functionalized high-AR droplets using maleimide-PEG5K-biotin. Following bioconjugation to the single cysteine residues of S28C, S28C-PEG-biotin was used to generate ellipsoidal droplets presenting biotin at the interface. To confirm availability and reactivity of this functional moiety, Alexa fluor® 488 streptavidin was used to label the interface (Fig. 7A). Confocal images of DiI-labelled (red-fluorescent) oil droplets demonstrated complete coverage of biotin at droplet surfaces, as indicated by the continuous fluorescent streptavidin ring around the droplet periphery (Fig. 7B). The droplets maintained the ellipsoidal shapes throughout the one-hour labelling process.

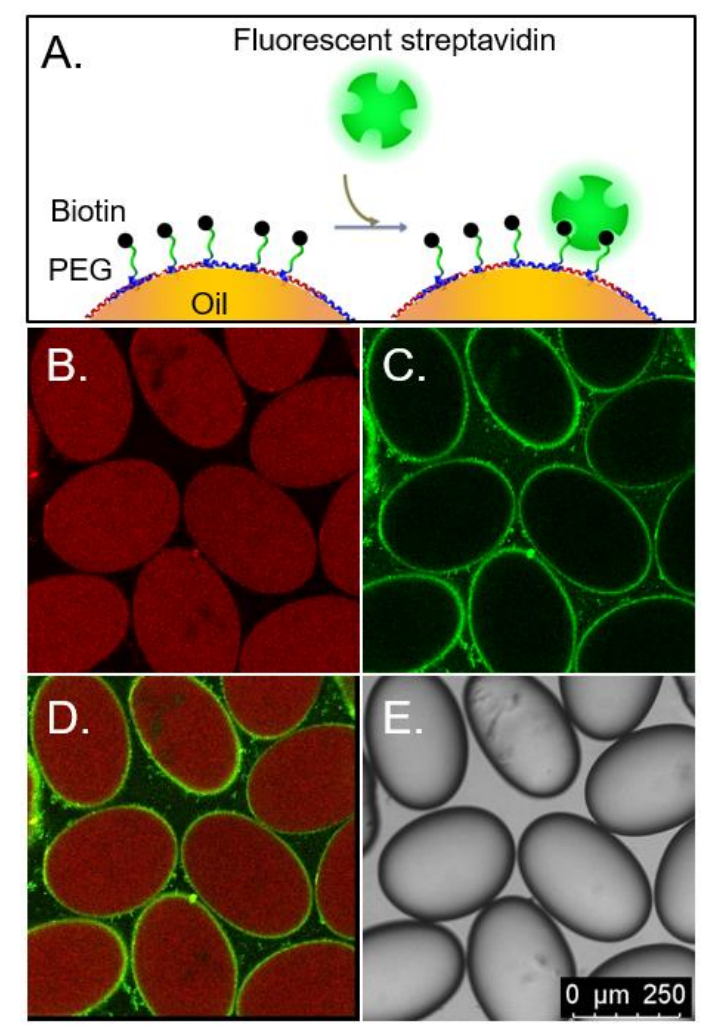

Figure 7. Biotin-functionalized droplets. (A) Schematic illustration showing fluorescent streptavidin binding onto biotin-functionalized droplet interface. (B) Confocal micrograph of DiI inside the oil droplets, (C) confocal micrograph of fluorescent streptavidin on the biotin-functionalized interface, (D) overlay of both confocal channels, and (E) corresponding brightfield image of the droplets. 


\section{CONCLUSION}

In summary, we present a microfluidic method for producing mono-dispersed oil-in-water emulsion droplets with non-spherical dimensions. We found that PEGylation of the protein S28C changed the mechanical properties of the protein film network, resulting in controllable droplet shape. Nonspherical oil droplets of a range of aspect ratios (from 1.0 to 3.4) can be produced by simply changing the flow rates within the designed channel. The range of droplet dimensions could feasibly be further increased with additional channel designs. The resulting droplet size and stability are discussed as a function of the flow rate ratios and mechanistic understanding of interfacial film properties. The droplets are stable against coalescence and the non-spherical shape can be maintained for several hours to days depending on the surface coverage of PEG molecule. This allows for topdown functionalization of non-spherical micro-droplets. Beyond this proof-of-concept study, further work will be aimed at: (1) quantitatively determining the relationship between macroscopic droplet shape-durability and corresponding microscopic structures at the interface; (2) investigating the possibility of producing molded shape-specific droplets by taking advantage of the 'shapememorable' property of the protein film; (3) using these non-spherical droplets as template for constructing anisotropic compartments for various applications, such as biochemical assays.

\section{ASSOCIATED CONTENT}

Supporting Information. The supporting information is available free of charge via the internet.

Protein conjugation procedures and characterization results by RP-HPLC and SDS-PAGE; two-inlet microfluidic device configuration; fitting to the modified model of elasticity effect on droplet size; illustration of Cambridge Interfacial Tensiometer setup and proposed explanations to different mechanical responses of protein films; calculation of supplied proteins to droplets; Initial IFT values. 


\section{AUTHOR INFORMATION}

Corresponding Author

* E-mail: z.chunxia@uq.edu.au(Chun-Xia Zhao)

* E-mail: f.sainsbury@griffith.edu.au(Frank Sainsbury)

ORCID

Yuan Gao: 0000-0002-7903-0311

Chun-Xia Zhao: 0000-0002-3365-3759

Frank Sainsbury: 0000-0001-8152-3820

Author Contributions

Y.G. performed the experiments, analyzed the data and drafted the manuscript, C.-X.Z. and F.S. help with designing the experiments, data analysis and manuscript revising. All authors have given approval to the final version of the manuscript.

Funding Sources

This research was supported by the Australian Research Council under Discovery Project (DP150100798). C.-X.Z. acknowledges financial support from the award of the Australian Research Council (ARC) Future Fellowship (FT140100726). F.S. acknowledges support from the Commonwealth Scientific and Industrial Research Organization (CSIRO) in the form of a Synthetic Biology Future Science Platform Fellowship.

Notes

The authors declare no conflicts of interest.

\section{ACKNOWLEDGMENT}

Y.G. acknowledges scholarships from the University of Queensland (UQ International scholarship). This work was performed in part at the Queensland node of the Australian National Fabrication Facility (ANFF-Q), a company established under the National Collaborative Research Infrastructure Strategy to provide nano and micro-fabrication facilities for Australia's researchers. 


\section{REFERENCES}

(1) Huebner, A.; Sharma, S.; Srisa-Art, M.; Hollfelder, F.; Edel, J. B.; deMello, A. J. Microdroplets: A sea of applications? Lab on a Chip 2008, 8 (8), 1244-1254, DOI: 10.1039/B806405A.

(2) Huebner, A.; Olguin, L. F.; Bratton, D.; Whyte, G.; Huck, W. T. S.; de Mello, A. J.; Edel, J. B.; Abell, C.; Hollfelder, F. Development of Quantitative Cell-Based Enzyme Assays in Microdroplets. Analytical Chemistry 2008, 80 (10), 3890-3896, DOI: 10.1021/ac800338z.

(3) Chiu, Y.-L.; Chan, H. F.; Phua, K. K. L.; Zhang, Y.; Juul, S.; Knudsen, B. R.; Ho, Y.-P.; Leong, K. W. Synthesis of Fluorosurfactants for Emulsion-Based Biological Applications. ACS Nano 2014, 8 (4), 3913-3920, DOI: 10.1021/nn500810n.

(4) Abalde-Cela, S.; Taladriz-Blanco, P.; de Oliveira, M. G.; Abell, C. Droplet microfluidics for the highly controlled synthesis of branched gold nanoparticles. Scientific Reports 2018, 8 (1), 2440, DOI: 10.1038/s41598-018-20754-x.

(5) Yang, Z.; Wei, J.; Sobolev, Y. I.; Grzybowski, B. A. Systems of mechanized and reactive droplets powered by multi-responsive surfactants. Nature 2018, 553 (7688), 313-318, DOI: 10.1038/nature25137.

(6) Chowdhury, M. S.; Zheng, W.; Kumari, S.; Heyman, J.; Zhang, X.; Dey, P.; Weitz, D. A.; Haag, R. Dendronized fluorosurfactant for highly stable water-in-fluorinated oil emulsions with minimal inter-droplet transfer of small molecules. Nature Communications 2019, 10 (1), 4546, DOI: 10.1038/s41467-019-12462-5.

(7) Song, H.; Chen, D. L.; Ismagilov, R. F. Reactions in Droplets in Microfluidic Channels. Angewandte Chemie International Edition 2006, 45 (44), 7336-7356, DOI: 10.1002/anie.200601554.

(8) Shojaeian, M.; Lehr, F.-X.; Göringer, H. U.; Hardt, S. On-Demand Production of Femtoliter Drops in Microchannels and Their Use as Biological Reaction Compartments. Analytical Chemistry 2019, 91 (5), 3484-3491, DOI: 10.1021/acs.analchem.8b05063.

(9) Gielen, F.; Butz, M.; Rees, E. J.; Erdelyi, M.; Moschetti, T.; Hyvönen, M.; Edel, J. B.; Kaminski, C. F.; Hollfelder, F. Quantitative Affinity Determination by Fluorescence Anisotropy Measurements of Individual Nanoliter Droplets. Analytical Chemistry 2017, 89 (2), 1092-1101, DOI: 10.1021/acs.analchem.6b02528.

(10) Taly, V.; Pekin, D.; Abed, A. E.; Laurent-Puig, P. Detecting biomarkers with microdroplet technology. Trends in Molecular Medicine 2012, 18 (7), 405-416, DOI: https://doi.org/10.1016/j.molmed.2012.05.001.

(11) Duraiswamy, S.; Khan, S. A. Droplet-Based Microfluidic Synthesis of Anisotropic Metal Nanocrystals. Small 2009, 5 (24), 2828-2834, DOI: 10.1002/smll.200901453.

(12) Zhang, H.; Tumarkin, E.; Peerani, R.; Nie, Z.; Sullan, R. M. A.; Walker, G. C.; Kumacheva, E. Microfluidic Production of Biopolymer Microcapsules with Controlled Morphology. Journal of the American Chemical Society 2006, 128 (37), 12205-12210, DOI: 10.1021/ja0635682.

(13) Fang, A.; Gaillard, C.; Douliez, J.-P. Template-Free Formation of Monodisperse DoughnutShaped Silica Microparticles by Droplet-Based Microfluidics. Chemistry of Materials 2011, 23 (21), 4660-4662, DOI: 10.1021/cm202145s.

(14) Kim, J. H.; Jeon, T. Y.; Choi, T. M.; Shim, T. S.; Kim, S.-H.; Yang, S.-M. Droplet Microfluidics for Producing Functional Microparticles. Langmuir 2014, 30 (6), 1473-1488, DOI: 10.1021/la403220p. 
(15) Yadavali, S.; Jeong, H.-H.; Lee, D.; Issadore, D. Silicon and glass very large scale microfluidic droplet integration for terascale generation of polymer microparticles. Nature Communications 2018, 9 (1), 1222, DOI: 10.1038/s41467-018-03515-2.

(16) Zeininger, L.; Weyandt, E.; Savagatrup, S.; Harvey, K. S.; Zhang, Q.; Zhao, Y.; Swager, T. M. Waveguide-based chemo- and biosensors: complex emulsions for the detection of caffeine and proteins. Lab on a Chip 2019, 19 (8), 1327-1331, DOI: 10.1039/C9LC00070D.

(17) Hall, P.; Seminara, G. Nonlinear oscillations of non-spherical cavitation bubbles in acoustic fields. Journal of Fluid Mechanics 2006, 101 (2), 423-444, DOI: 10.1017/S0022112080001735.

(18) Hwang, D. K.; Dendukuri, D.; Doyle, P. S. Microfluidic-based synthesis of non-spherical magnetic hydrogel microparticles. Lab on a Chip 2008, 8 (10), 1640-1647, DOI: 10.1039/B805176C.

(19) Liu, X.; Kent, N.; Ceballos, A.; Streubel, R.; Jiang, Y.; Chai, Y.; Kim, P. Y.; Forth, J.; Hellman, F.; Shi, S.; Wang, D.; Helms, B. A.; Ashby, P. D.; Fischer, P.; Russell, T. P. Reconfigurable ferromagnetic liquid droplets. Science 2019, 365 (6450), 264, DOI: 10.1126/science.aaw8719.

(20) Nagelberg, S.; Zarzar, L. D.; Nicolas, N.; Subramanian, K.; Kalow, J. A.; Sresht, V.; Blankschtein, D.; Barbastathis, G.; Kreysing, M.; Swager, T. M.; Kolle, M. Reconfigurable and responsive droplet-based compound micro-lenses. Nature Communications 2017, 8 (1), 14673, DOI: 10.1038/ncomms 14673 .

(21) Leal-Calderon, F.; Schmitt, V. Solid-stabilized emulsions. Current Opinion in Colloid \& Interface Science 2008, 13 (4), 217-227, DOI: https://doi.org/10.1016/j.cocis.2007.09.005.

(22) Tenjimbayashi, M.; Higashi, M.; Yamazaki, T.; Takenaka, I.; Matsubayashi, T.; Moriya, T.; Komine, M.; Yoshikawa, R.; Manabe, K.; Shiratori, S. Droplet Motion Control on Dynamically Hydrophobic Patterned Surfaces as Multifunctional Liquid Manipulators. ACS Applied Materials \& Interfaces 2017, 9 (12), 10371-10377, DOI: 10.1021/acsami.7b01641.

(23) Raj, R.; Adera, S.; Enright, R.; Wang, E. N. High-resolution liquid patterns via three-dimensional droplet shape control. Nature Communications 2014, 5, 4975, DOI: 10.1038/ncomms5975

(24) Hancock, M. J.; Yanagawa, F.; Jang, Y.-H.; He, J.; Kachouie, N. N.; Kaji, H.; Khademhosseini, A. Designer Hydrophilic Regions Regulate Droplet Shape for Controlled Surface Patterning and 3D Microgel Synthesis. Small 2012, 8 (3), 393-403, DOI: 10.1002/smll.201101745.

(25) Ennis, J. Spontaneous curvature of surfactant films. The Journal of Chemical Physics 1992, 97 (1), 663-678, DOI: 10.1063/1.463561.

(26) Kaufman, G.; Liu, W.; Williams, D. M.; Choo, Y.; Gopinadhan, M.; Samudrala, N.; Sarfati, R.; Yan, E. C. Y.; Regan, L.; Osuji, C. O. Flat Drops, Elastic Sheets, and Microcapsules by Interfacial Assembly of a Bacterial Biofilm Protein, BslA. Langmuir 2017, 33 (47), 13590-13597, DOI: 10.1021/acs.langmuir.7b03226.

(27) Liu, K.; Ding, H.-J.; Liu, J.; Chen, Y.; Zhao, X.-Z. Shape-Controlled Production of Biodegradable Calcium Alginate Gel Microparticles Using a Novel Microfluidic Device. Langmuir 2006, 22 (22), 9453-9457, DOI: 10.1021/la061729+.

(28) Xu, S.; Nie, Z.; Seo, M.; Lewis, P.; Kumacheva, E.; Stone, H. A.; Garstecki, P.; Weibel, D. B.; Gitlin, I.; Whitesides, G. M. Generation of Monodisperse Particles by Using Microfluidics: Control over Size, Shape, and Composition. Angewandte Chemie International Edition 2005, 44 (5), 724-728, DOI: 10.1002/anie.200462226.

(29) Ono, T.; Yamada, M.; Suzuki, Y.; Taniguchi, T.; Seki, M. One-step synthesis of spherical/nonspherical polymeric microparticles using non-equilibrium microfluidic droplets. RSC Advances 2014, 4 (26), 13557-13564, DOI: 10.1039/C4RA00670D. 
(30) Nisisako, T.; Hatsuzawa, T. A microfluidic cross-flowing emulsion generator for producing biphasic droplets and anisotropically shaped polymer particles. Microfluidics and Nanofluidics 2010, 9 (2), 427-437, DOI: 10.1007/s10404-009-0559-6.

(31) Dexter, A. F.; Malcolm, A. S.; Middelberg, A. P. J. Reversible active switching of the mechanical properties of a peptide film at a fluid-fluid interface. Nature Materials 2006, 5 (6), 502-506, DOI: $10.1038 / \mathrm{nmat} 1653$.

(32) Tayeb, H. H.; Stienecker, M.; Middelberg, A. P. J.; Sainsbury, F. Impact of Site-Specific Bioconjugation on the Interfacial Activity of a Protein Biosurfactant. Langmuir 2019, 35 (42), 13588-13594, DOI: 10.1021/acs.langmuir.9b01684.

(33) Tayeb, H. H.; Piantavigna, S.; Howard, C. B.; Nouwens, A.; Mahler, S. M.; Middelberg, A. P. J.; He, L.; Holt, S. A.; Sainsbury, F. Insights into the interfacial structure-function of poly(ethylene glycol)-decorated peptide-stabilised nanoscale emulsions. Soft Matter 2017, 13 (43), 7953-7961, DOI: 10.1039/C7SM01614J.

(34) Middelberg, A. P. J.; Dimitrijev-Dwyer, M. A Designed Biosurfactant Protein for Switchable Foam Control. ChemPhysChem 2011, 12 (8), 1426-1429, DOI: 10.1002/cphc.201100082.

(35) Caen, O.; Schütz, S.; Jammalamadaka, M. S. S.; Vrignon, J.; Nizard, P.; Schneider, T. M.; Baret, J.-C.; Taly, V. High-throughput multiplexed fluorescence-activated droplet sorting. Microsystems \& Nanoengineering 2018, 4 (1), 33, DOI: 10.1038/s41378-018-0033-2.

(36) Zhao, C. X.; Middelberg, A. P. J. One-step fabrication of titania hollow spheres by controlled interfacial reaction in a droplet-based microfluidic system. Microfluidics and Nanofluidics 2013, 14 (3-4), 703-709, DOI: 10.1007/s10404-012-1088-2.

(37) Zhao, C. X.; Middelberg, A. P. J. Titania microparticles using a facile microfluidic mass-transfer control method. Chemical Engineering Science 2014, 112, 10-14, DOI: 10.1016/j.ces.2014.03.009.

(38) Middelberg, A. P. J.; He, L.; Dexter, A. F.; Shen, H. H.; Holt, S. A.; Thomas, R. K. The interfacial structure and Young's modulus of peptide films having switchable mechanical properties. Journal of The Royal Society Interface 2008, 5 (18), 47-54, DOI: 10.1098/rsif.2007.1063.

(39) de Bruin, M. G. In Simultaneous pade approximation and orthogonality, Polynômes Orthogonaux et Applications, Berlin, Heidelberg, 1985//; Brezinski, C.; Draux, A.; Magnus, A. P.; Maroni, P.; Ronveaux, A., Eds. Springer Berlin Heidelberg: Berlin, Heidelberg, 1985; pp 74-83.

(40) He, Q.; Zhang, J.; Shi, J.; Zhu, Z.; Zhang, L.; Bu, W.; Guo, L.; Chen, Y. The effect of PEGylation of mesoporous silica nanoparticles on nonspecific binding of serum proteins and cellular responses. Biomaterials 2010, 31 (6), 1085-1092, DOI: 10.1016/j.biomaterials.2009.10.046.

(41) Baroud, C. N.; Gallaire, F.; Dangla, R. Dynamics of microfluidic droplets. Lab on a Chip 2010, 10 (16), 2032-2045, DOI: 10.1039/C001191F.

(42) Zhu, P.; Wang, L. Passive and active droplet generation with microfluidics: a review. Lab on $a$ Chip 2017, 17 (1), 34-75, DOI: 10.1039/C6LC01018K.

(43) Zhao, C.-X.; Miller, E.; Cooper-White, J. J.; Middelberg, A. P. J. Effects of fluid-fluid interfacial elasticity on droplet formation in microfluidic devices. AIChE Journal 2011, 57 (7), 1669-1677, DOI: $10.1002 /$ aic. 12382.

(44) Zhao, C.-X.; Rondeau, E.; Cooper-White, J. J.; Middelberg, A. P. J. Microfluidic Elucidation of the Effects of Interfacial Rheology on Droplet Deformation. Industrial \& Engineering Chemistry Research 2012, 51 (4), 2021-2029, DOI: 10.1021/ie200631m.

(45) Korczyk, P. M.; van Steijn, V.; Blonski, S.; Zaremba, D.; Beattie, D. A.; Garstecki, P. Accounting for corner flow unifies the understanding of droplet formation in microfluidic channels. Nature Communications 2019, 10 (1), 2528, DOI: 10.1038/s41467-019-10505-5. 
(46) Vladisavljević, G. T.; Duncanson, W. J.; Shum, H. C.; Weitz, D. A. Emulsion Templating of Poly(lactic acid) Particles: Droplet Formation Behavior. Langmuir 2012, 28 (36), 12948-12954, DOI: 10.1021/la302092f.

(47) Qian, B.; Shi, S.; Wang, H.; Russell, T. P. Reconfigurable Liquids Stabilized by DNA Surfactants. ACS Applied Materials \& Interfaces 2020, 12 (11), 13551-13557, DOI: 10.1021/acsami.0c01487.

(48) Saïdi, A.; Martin, C.; Magnin, A. Influence of yield stress on the fluid droplet impact control. Journal of Non-Newtonian Fluid Mechanics 2010, 165 (11), 596-606, DOI: https://doi.org/10.1016/j.jnnfm.2010.02.020.

(49) Bartolo, D.; Boudaoud, A.; Narcy, G.; Bonn, D. Dynamics of Non-Newtonian Droplets. Physical Review Letters 2007, 99 (17), 174502, DOI: 10.1103/PhysRevLett.99.174502.

(50) Krägel, J.; Derkatch, S. R.; Miller, R. Interfacial shear rheology of protein-surfactant layers. Advances in Colloid and Interface Science 2008, 144 (1), 38-53, DOI: https://doi.org/10.1016/j.cis.2008.08.010.

(51) Cao, C.; Zhou, Z.-L.; Zheng, L.; Huang, Q.-L.; Du, F.-P. Dilational rheology of different globular protein with imidazolium-based ionic liquid surfactant adsorption layer at the decane/water interface. Journal of Molecular Liquids 2017, 233, 344-351, DOI: https://doi.org/10.1016/j.molliq.2017.02.121.

(52) Lim, H.; Hoag, S. W. Plasticizer Effects on Physical-Mechanical Properties of Solvent Cast Soluplus ${ }^{\circledR}$ Films. AAPS PharmSciTech 2013, 14 (3), 903-910, DOI: 10.1208/s12249-013-9971$\mathrm{z}$.

(53) Dimitrijev-Dwyer, M.; Middelberg, A. P. J. The extensional viscoelasticity of protein-coated interfaces. Soft Matter 2011, 7 (17), 7772-7781, DOI: 10.1039/C1SM05253E.

(54) Knoche, S.; Vella, D.; Aumaitre, E.; Degen, P.; Rehage, H.; Cicuta, P.; Kierfeld, J. Elastometry of Deflated Capsules: Elastic Moduli from Shape and Wrinkle Analysis. Langmuir 2013, 29 (40), 12463-12471, DOI: 10.1021/la402322g.

(55) Jahnke, K.; Weiss, M.; Frey, C.; Antona, S.; Janiesch, J.-W.; Platzman, I.; Göpfrich, K.; Spatz, J. P. Programmable Functionalization of Surfactant-Stabilized Microfluidic Droplets via DNATags. Advanced Functional Materials 2019, 29 (23), 1808647, DOI: 10.1002/adfm.201808647.

(56) Zeng, B.; Chuan, Y.; O'Sullivan, B.; Caminschi, I.; Lahoud, M.; Thomas, R.; Middelberg, A. Receptor-Specific Delivery of Protein Antigen to Dendritic Cells by a Nanoemulsion Formed Using Top-Down Non-Covalent Click Self-Assembly. Small 2013, 9 (22), 3736-3742.

(57) Nguyen, N.; Lassemono, S.; Chollet, F. A. In Optical detection for droplet size control in microfluidic droplet-based analysis systems, The 13th International Conference on Solid-State Sensors, Actuators and Microsystems, 2005. Digest of Technical Papers. TRANSDUCERS '05., 5-9 June 2005; 2005; pp 1557-1560 Vol. 2. 
TOC:

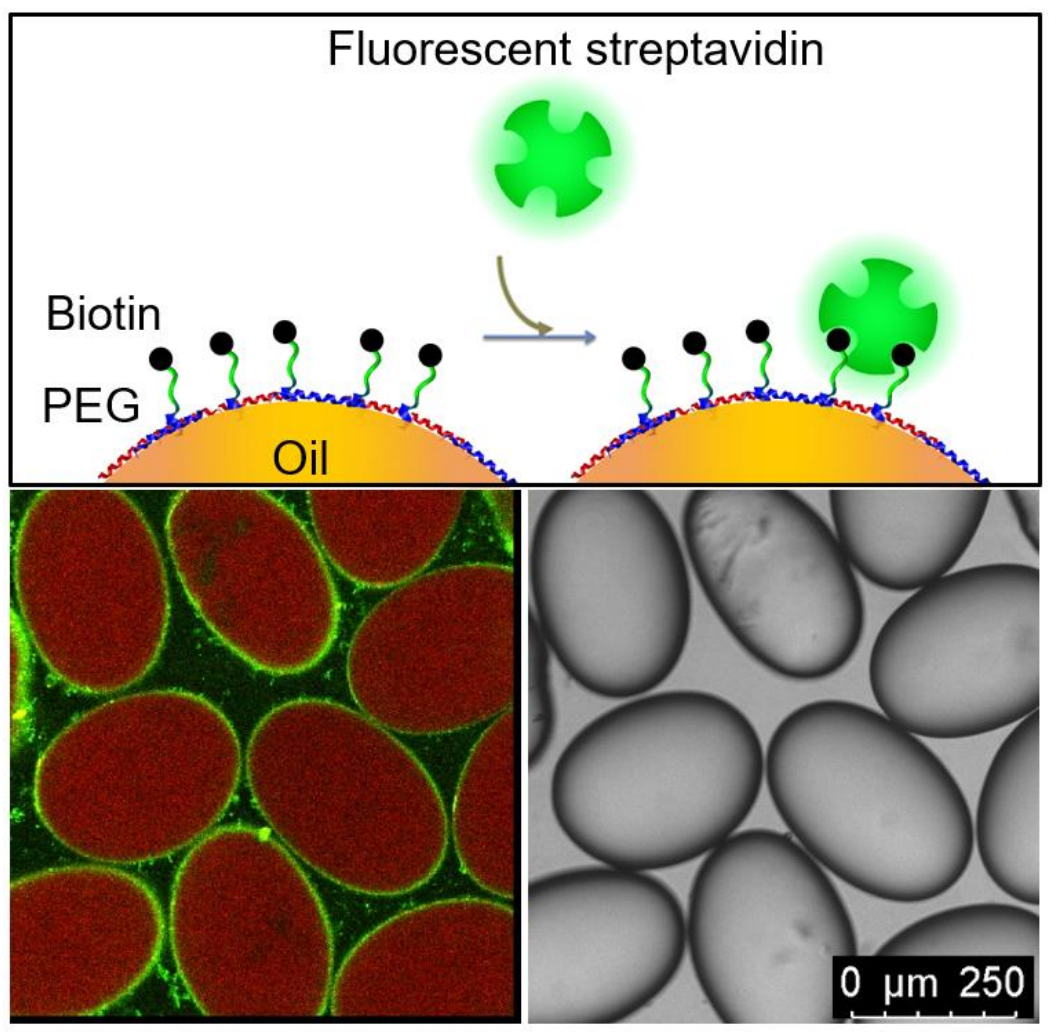




\section{Supporting Information}

\section{Droplet Shape Control Using Microfluidics and Designer Biosurfactants}

Yuan $\mathrm{Gao}^{1}$, Chun-Xia Zhao ${ }^{1 *}$, Frank Sainsbury ${ }^{1,2,3^{*}}$

${ }^{1}$ Australian Institute for Bioengineering and Nanotechnology, University of Queensland, St Lucia, QLD, 4072, Australia.

${ }^{2}$ Centre for Cell Factories and Biopolymers, Griffith Institute for Drug Discovery, Griffith University, Nathan, QLD, 4111, Australia.

${ }^{3}$ Synthetic Biology Future Science Platform, Commonwealth Scientific and Industrial Research Organization (CSIRO), Brisbane, QLD 4001, Australia. 

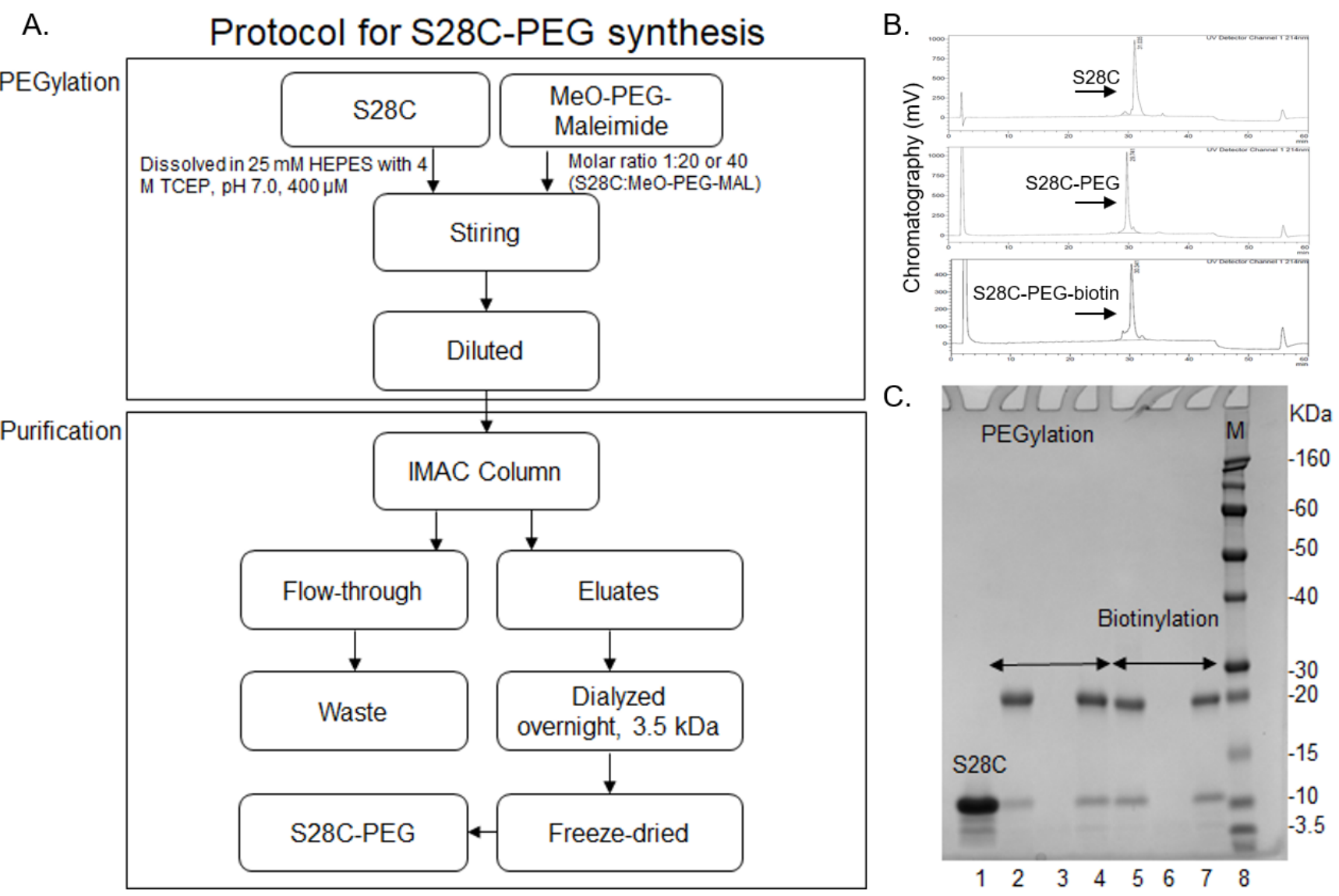

Figure S1. S28C conjugation procedures (A) and purified products checked by RP-HPLC (B) and SDS-PAGE (C). Conditions for Biotinylation are the same as PEGylation. For the SDS-PAGE, lane 1 is pure S28C protein, lane 2-4 and 5-7 are conjugation products, IMAC flow-throughs and eluates, for PEGylation and Biotinylation, respectively. Lane 8 is standard protein marker. 


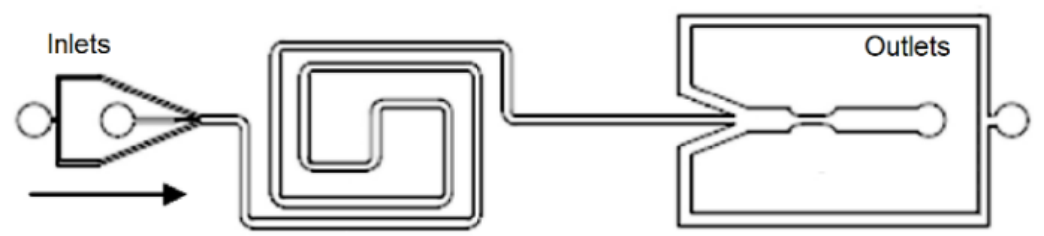

Figure S2. Microfluidic chip for rheological studies. Two inlets channel with a flow-focusing configuration was used, oil solution was introduced from the central channel (width: $40 \mu \mathrm{m}$ ), surfactant solutions were fed through the Y-shaped side channel. The oil phase was sheared by the aqueous surfactant phase at the junction and then entered a long spiral channel (width: $400 \mu \mathrm{m})$. The channel is $150 \mu \mathrm{m}$ in depth. 


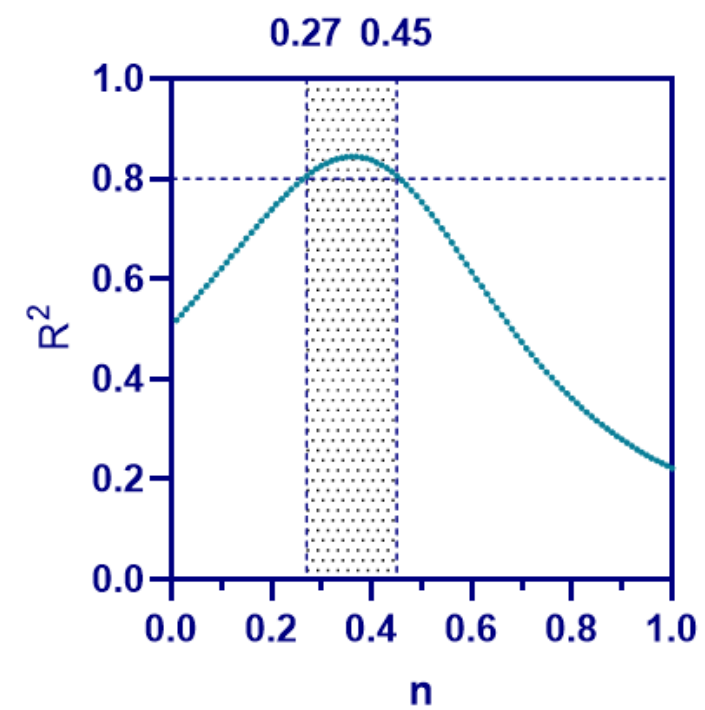

Figure S3. Fitness of droplet size to the proposed $(\sigma \cdot E)^{\mathrm{n}}$ modified model under different values of $n$. $\sigma$ is the interfacial tension, $\mathrm{E}$ is the elasticity factor, $\mathrm{n}$ is a constant. The shaded area indicates the $\mathrm{n}$ range $(0.36 \pm 0.09)$ that gives a good fitting of droplet sizes to the model $\mathrm{D} \propto\left(\mathrm{Q}_{\mathrm{d}} / \mathrm{Q}_{\mathrm{c}}\right)^{1 / 2} \cdot(\sigma \cdot \mathrm{E})^{0.36 \pm 0.09}$, with all $\mathrm{R}^{2}$ larger than 0.80 , when $\mathrm{n}=0.36, \mathrm{R}^{2}=0.84$.

A MATLAB script was used to determine the parameter $\mathrm{n}$. The normalized droplet sizes obtained under different surfactant solutions were divided by a correcting factor $(\sigma \cdot E)^{\mathrm{n}}$ to integrate the impact of surfactant-film elasticity on resulted droplet size. thus, re-scattered the droplet size distribution. Then a least square linear regression was applied to the re-scattered sizes to test if the data could be unified into certain function of $\left(\mathrm{Q}_{\mathrm{d}} / \mathrm{Q}_{\mathrm{c}}\right)^{1 / 2} . \mathrm{n}$ values ranging from 0 to 1 were swept at a step of 0.01 by a loop function in MATLAB, and the corresponding fitting R-square $\left(\mathrm{R}^{2}\right)$ were calculated. For $\mathrm{n}$ values within the range of $0.27-0.45$, the droplet sizes display a good linear fitting with $\left(\mathrm{Q}_{\mathrm{d}} / \mathrm{Q}_{\mathrm{c}}\right)^{1 / 2} \cdot(\sigma \cdot \mathrm{E})^{\mathrm{n}}$, with $\mathrm{R}^{2}>0.80$. 


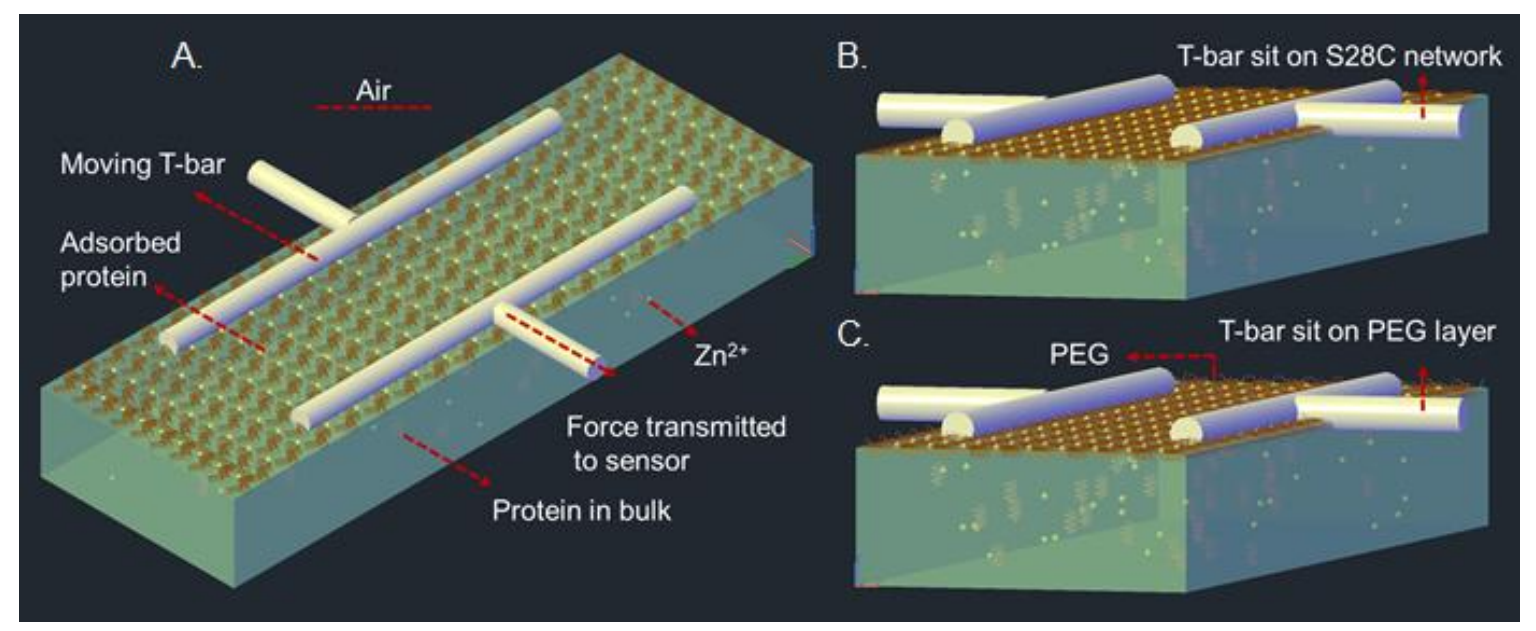

Figure S4. Illustration of Cambridge interfacial tensiometry (CIT) setup (A), showing the possible cause of different mechanical response of S28C (B) and S28C-PEG (C) protein networks. The biosurfactant solutions were added into the CIT trough, forming an interfacial layer between the two T-bars of the CIT apparatus. Pulling of T-bar transmits the force generated by interactions between the T-bar and interfacial protein network, where the T-bar slips on the PEG layer at the interface. Figures are not presented to scale. 

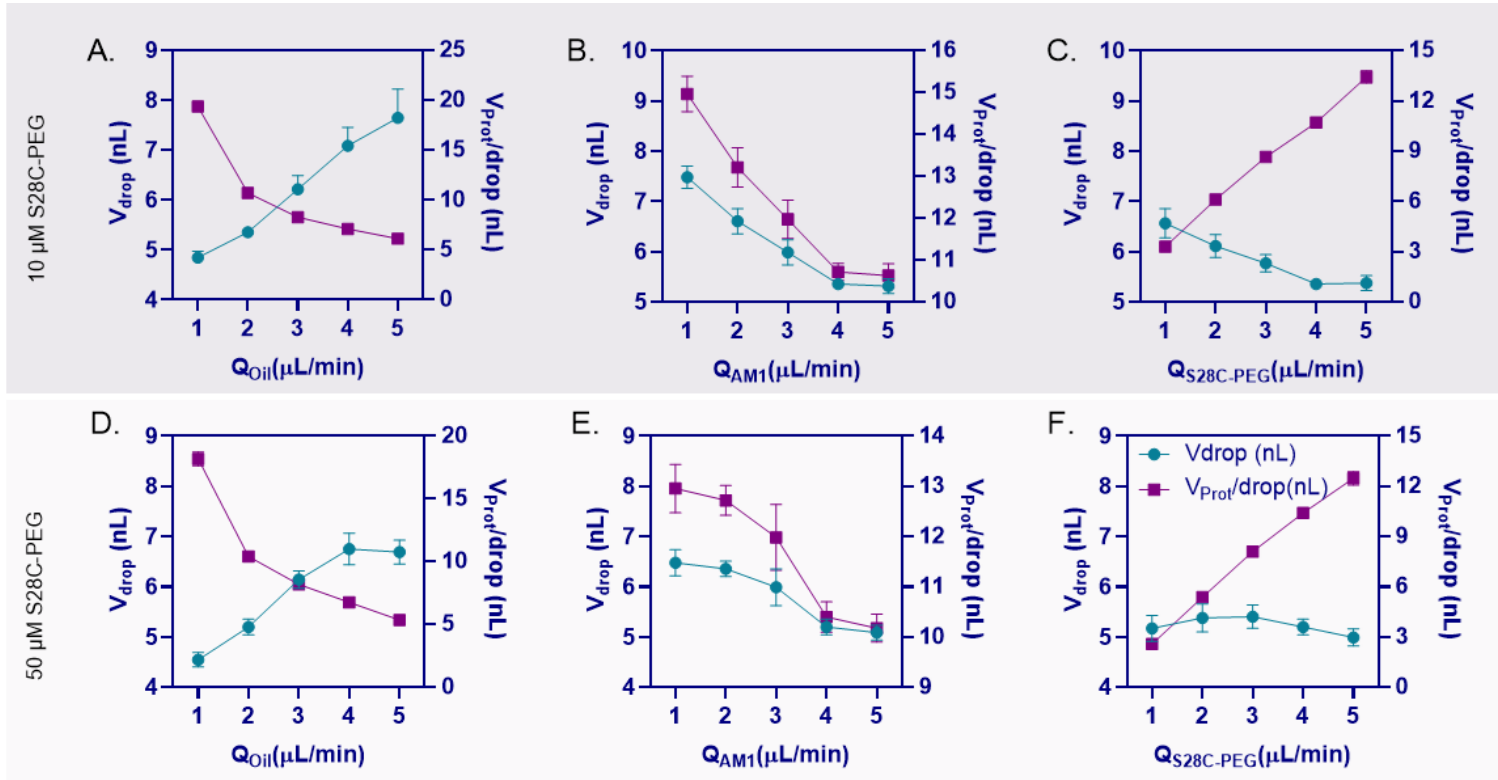

Figure S5. Generated droplet volume $(\bullet)$ and S28C-PEG volume supplied to each droplet ( $\bullet$ ) as a function of varying flow rates of (A) oil, (B) AM1 (C) S28C-PEG using $10 \mu \mathrm{M}$ S28C-PEG solutions and (D)-(F) are corresponding data for $50 \mu \mathrm{M}$ S28C-PEG solutions. Droplet volume was approximated by treating the droplets as ellipsoidal shapes. Droplet generation frequency was estimated by dividing the overall supplied oil volume by the resulted droplet volume ${ }^{57}$ : Freq $=$ $V_{\text {oil }} / V_{\text {drop }}$. Where $\mathrm{V}_{\text {oil }}$ is the total volume of supplied oil in one second, $\mathrm{V}_{\text {drop }}$ is the volume of the

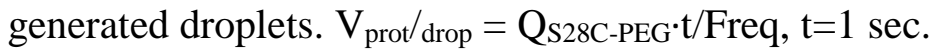


Table S1. Initial IFT values detected by the DSA apparatus was listed in this table as the transient interfacial tension for interfaces formed at microfluidic junction.

\begin{tabular}{lcccccc}
\hline & SDS & AM1 & S28C & $\begin{array}{l}\text { S28C- } \\
\text { PEG }\end{array}$ & AM1+S28C & $\begin{array}{l}\text { AM1+S28C- } \\
\text { PEG }\end{array}$ \\
\hline $\begin{array}{l}\text { Concentration } \\
(\mu \mathrm{M})\end{array}$ & 50 & 50 & 10 & 10 & $50+10$ & $50+10$ \\
IFT $\left(\mathrm{mN} \cdot \mathrm{m}^{-1}\right)$ & 2.80 & 14.10 & 17.06 & 21.36 & 15.82 & 14.71 \\
\hline
\end{tabular}

